\title{
Ionization in a 1-Dimensional Dipole Model
}

\author{
C. Stucchio, O. Costin and J. L. Lebowitz
}

September 25, 2018

\begin{abstract}
We study the evolution of a one dimensional model atom with $\delta$ function binding potential, subjected to a dipole radiation field $E(t) x$ with $E(t)$ a $2 \pi / \omega$-periodic real-valued function. Starting with an initially localized state and $E(t)$ a trigonometric polynomial, complete ionization occurs (the probability of finding the electron in any fixed region goes to zero).

For more general periodic fields and $\psi(x, 0)$ compactly supported (this is a technical point making the exposition cleaner), we construct a resonance expansion. More precisely, we prove that $\psi(x, t)$ has a unique decomposition into a quasi-bound state $e^{-i \sigma_{b} t} \psi_{b}(x, t)$ and a dispersive component $\psi_{d}(x, t)$ (both square integrable in space, with $\sigma_{b}$ and $\psi_{b}(x, t)$ independent of $\psi(x, 0)$ ). The quasi-bound state $\psi_{b}(x, t)$ is $2 \pi / \omega$ periodic in time and exponentially decaying in space. The dispersive part is given by a Borel summable asymptotic power series in $t^{-1 / 2}$ with coefficients varying with $x$. In the event $\Im \sigma_{b}=0$, then $\psi_{b}(x, t)$ is a Floquet eigenstate and orthogonal to $\psi_{d}(x, t)$.
\end{abstract}

\section{Introduction}

The ionization of an atom by an electromagnetic field is one of the central problems of atomic physics. Despite this, there are few exact results available for the ionization of a bound particle by a realistic time-periodic electric field of the dipole form $\vec{E}(t) \cdot \vec{x}$ (an AC-Stark field) for fields of arbitrary strength. The most realistic results we are aware of are based on complex scaling (18, 31) and show ionization of certain bound states of the Coulomb atom as well as defining resonances for small electric field.

The lack of rigorous results for large electric field is true not only for realistic systems with Coulombic binding potential, but even for model systems with short range binding potential [3, 5, 17. The most idealized version of the latter has an attractive $\delta$-function potential in 1 dimension. Its spectrum consists of just one bound state plus the continuum [10. This model, in which all states are explicitly known, has been studied extensively in the literature. Even for this simple model, the only rigorous results (known to us) concerning ionization involve short range external forcing potentials rather than the dipole interaction; 
see however 4, 16, 21] for some rigorous bounds on the ionization probability by a dipole potential for finite time pulses. In this paper we extend the results of [9, 10, 26] to the case of dipole interactions.

We consider the time evolution of a particle in one dimension governed by the Schrödinger equation (in appropriate units):

$$
\begin{aligned}
i \partial_{t} \psi(x, t) & =\left(-\frac{\partial^{2}}{\partial x^{2}}-2 \delta(x)\right) \psi(x, t)+E(t) x \psi(x, t) \\
\psi(x, 0) & =\psi_{0}(x) \in L^{2}(\mathbb{R})
\end{aligned}
$$

Here, $(x, t) \in \mathbb{R} \times \mathbb{R}^{+}, E(t)$ is real valued, smooth and $E(t)=E(t+2 \pi / \omega)$. Taking $\psi_{0}(x)$ initially localized state (e.g. $\psi_{0}(x)=e^{-|x|}$, the bound state of $\left.H_{0}=-\partial_{x}^{2}-2 \delta(x)\right)$, we prove that for $E(t)$ a non-zero trigonometric polynomial,

$$
E(t)=\sum_{n=1}^{N}\left(E_{n} e^{i n \omega t}+\overline{E_{n}} e^{-i n \omega t}\right)
$$

the system always ionizes. That is, for $\psi(x, t)$ solving (1.1),

$$
\lim _{t \rightarrow \infty} \int_{-L}^{L}|\psi(x, t)|^{2} d x=0, \quad \forall L \in \mathbb{R}^{+}
$$

with the approach to zero at least as fast as $O\left(t^{-1}\right)$.

We go further than this and prove that for compactly supported initial data, $\psi(x, t)$ can be uniquely decomposed into a sum of $L^{2}(\mathbb{R}, d x)$ functions, $\psi(x, t)=$ $e^{-i \sigma_{b} t} \psi_{b}(x, t)+\psi_{d}(x, t)$. The function $\psi_{b}(x, t)$ is $2 \pi / \omega$ periodic in time and exponentially decaying in space, and $e^{-i \sigma_{b} t} \psi_{b}(x, t)$ corresponds to a quasi-bound state. Note that $\Im \sigma_{b} \leq 0$, since otherwise unitary evolution would be violated. The term $\psi_{d}(x, t)$ has a Borel summable transseries expansion in time with power law terms in $t^{-n / 2}$ for $n \geq 1$. In fact, letting

$$
\gamma=-\Im \sigma_{b}
$$

then for small values of $\gamma, 2 \gamma$ gives the dominant part of the ionization rate for most experimentally relevant times $[3$. The ionization rate $2 \gamma$ will always be small when the strength of the radiation field is small.

The term $\psi_{d}(x, t)$ has a Borel summable transseries expansion in time with power law terms in $t^{-n / 2}$ for $n \geq 1$, which allows the unique definition of $\psi_{b}(x, t)$. We note that the polynomially decaying component of of the wavefunction has actually been observed experimentally in quantum systems [27], although under significantly different ${ }^{1}$ physical conditions.

Furthermore, setting $E(t)=\epsilon E(t)$, one can show that $\sigma_{b}$ and $\psi_{b}(x, t)$ have convergent power series expansions in $\epsilon$ when $\omega^{-1} \notin \mathbb{N}$. When $\epsilon \rightarrow 0$, $e^{-i \sigma_{b} t} \psi_{b}(x, t) \rightarrow e^{i t} e^{-|x|}$, the bound state of $H_{0}$ and $\psi_{d}(x, t)$ goes to the projection of $\psi(x, t)$ on the continuum states of $H_{0}$. This shows that the resonance is

\footnotetext{
${ }^{1}$ In [27, they studied luminescence decay of dissolved organic materials after pulsed laser excitation.
} 
the analytic continuation, in $\epsilon$, of the bound state. The Fermi golden rule and multiphoton generalizations can be recovered by doing perturbation theory in our formalism. This will be presented (in greater generality) in a separate work.

When $E(t)$ is not a trigonometric polynomial, the Floquet Hamiltonian (see below) may have time dependent bound states, and ionization may fail. This is uncommon, but there are examples of time periodic Schrödinger operators for which there exist such bound states [10, 24, 26].

Our results are restricted to the one dimensional case. We believe, however, that these results can be extended without too much difficulty to the three dimensional case with a $\delta$-function binding potential (see Section 4.3). We also believe that the techniques used here can be used for quantitative calculations in realistic physical problems, although this is not done here.

\subsection{Outline of the strategy}

Due to the fact that the binding potential $\delta(x)$ has support $\{0\}$, the behavior of $\psi(0, t)$ and the initial condition completely determine the behavior of the solution.

Our main tool is the study of the analytic structure of the Zak transform of $\psi(0, t)$ (with $\psi(0, t)=0$ for $t<0)$,

$$
\mathcal{Z}[\psi(0, \cdot)](\sigma, t)=\sum_{j \in \mathbb{Z}} e^{i \sigma(t+2 \pi j / \omega)} \psi(0, t+2 \pi j / \omega)
$$

in the complex $\sigma$ domain. It is sufficient to consider the strip $0 \leq \Re \sigma<\omega$, which we shall do henceforth (see Definition 3.1 for an explanation of why). It is also sufficient to consider only $x=0$ since that is the support of the $\delta$ function binding potential. Unitary evolution of the wavefunction implies that $\mathcal{Z}[\psi(0, \cdot)](\sigma, t)$ is analytic in $\sigma$ for $\Im \sigma>0$.

For $E(t)=0$ and $\left\langle\psi(x, 0) \mid e^{-|x|}\right\rangle \neq 0, \mathcal{Z}[\psi(0, \cdot)](\sigma, t)$ has a pole at $\sigma_{b}=-1+$ $\lfloor 1 / \omega\rfloor \omega$ corresponding to the eigenvalue -1 of the unperturbed Hamiltonian. The residue at the pole is $e^{-i\lfloor 1 / \omega\rfloor \omega t}$. If we consider $\sigma$ outside the strip $0 \leq$ $\Re \sigma<\omega$, we will find this pole repeated at the points $\sigma_{b}+m \omega$ (see Definition 3.1 in particular (3.6c)).

When $E \neq 0$, the pole gives rise to the term $e^{-i \sigma_{b} t} \psi_{b}(x, t)$, with the residue at the poles corresponding (by a linear transformation) to the Fourier coefficients in time of $\psi_{b}(x, t)$. There is also a branch point at $\sigma=0$ which gives rise to the dispersive part of the wavefunction.

The proof of complete ionization, (1.3), involves proving that any Floquet bound state (solution to (1.8), below) must be zero. This is done by solving the Schrodinger equation without the $\delta$-function at zero, and showing that solutions which decay exponentially as $x \rightarrow-\infty$ can not be matched continuously at $x=0$ to solutions which decay exponentially as $x \rightarrow+\infty$. This consequently implies that $\Im \sigma<0$. 


\subsection{Statement of results}

We consider the Schrödinger equation with a time periodic Stark Hamiltonian (1.1) on $\mathbb{R}^{1+1}$. $E(t)$ is given by (1.2) for some set of $E_{n}$ (at least one of which is not zero).

Subject to these assumptions, we prove two theorems:

Theorem 1 Suppose $\psi_{0}(x)$ is compactly supported and in $H^{1}$ (finite kinetic energy). Then the wave function $\psi(x, t)$, the solution of (1.1), can be decomposed uniquely into a quasi-bound state (or bound state, if $\Im \sigma_{b}=0$ ) and a dispersive part $\psi_{d}(x, t)$ :

$$
\psi(x, t)=e^{-i \sigma_{b} t} \psi_{b}(x, t)+\psi_{d}(x, t)
$$

where $\Im \sigma_{b} \leq 0, \psi_{b}(x, t)$ is $2 \pi / \omega$ periodic in time, and for each $t, \psi_{b}(x, t) \in$ $H^{1}(\mathbb{R})$. In particular $\Im \sigma_{b}$ is uniquely determined by $E(t)$, and $\psi_{b}(x, t)$ can vary at most by a constant factor. The quasimode $\psi_{b}(x, t)$ decays exponentially in space, i.e. $\left|\psi_{b}(x, t)\right| \leq C e^{-\alpha|x|}$ with $\alpha$ given by:

$$
\alpha=\inf _{n \in \mathbb{Z}}\left|\Im \sqrt{\sigma_{b}+n \omega}\right|
$$

When $\sigma_{b} \in(0, \omega)$, then $\psi_{b}(x, t)$ is a Floquet bound state. That is, it is a continuous, nonzero solution of the Floquet equation:

$$
\begin{gathered}
\left(-i \partial_{t}-\frac{\partial^{2}}{\partial x^{2}}-2 \delta(x)+E(t) x\right) \psi_{b}(x, t)=\sigma_{b} \psi_{b}(x, t) \\
\lim _{x \rightarrow-\infty} \psi_{b}(x, t)=\lim _{x \rightarrow \infty} \psi_{b}(x, t)=0 \\
\psi_{b}(x, t)=\psi_{b}(x, t+2 \pi / \omega)
\end{gathered}
$$

In this case, the functions $\psi_{b}(x, t)$ and $\psi_{d}(x, t)$ are orthogonal, that is $\left\langle\psi_{b}(x, t) \mid \psi_{d}(x, t)\right\rangle_{L^{2}(\mathbb{R}, d x)}=0$. Finally, $\psi_{d}(x, t)$ is Borel summable, i.e.:

$$
\psi_{d}(x, t)=\sum_{j \in \mathbb{Z}} e^{i j \omega t} \mathcal{L} \mathcal{B} \sum_{n=3}^{\infty} D_{j, n}(x) t^{-n / 2}
$$

where $\mathcal{L B}$ is the Borel summation operator, see [7, 15].

Theorem 2 (Ionization) Suppose $E(t)$ is a trigonometric polynomial, i.e. $E_{n}=0$ for $n>N$. Then ionization occurs:

$$
\lim _{t \rightarrow \infty} \int_{-L}^{L}|\psi(x, t)|^{2} d x=0
$$

If $\psi_{0}(x) \in L^{1}(\mathbb{R}) \cap L^{2}\left(\mathbb{R}\right.$, then the approach to zero is at least as fast as $t^{-1}$. If in addition $\psi_{0}(x)$ is compactly supported, then the decay exponent $\gamma=-\Im \sigma_{b}$ is strictly positive (cf. (1.4), (1.6)). 
Remark 1.1 The PDE (1.8) is formally overdetermined, since it has 4 boundary conditions (1.8b) and (1.8c) ). This makes nonzero solutions to (1.8) unlikely, although there may be some special forms of $E(t)$ for which such a solution can be found. The proof of Theorem 2 is essentially a proof that in the case of $E(t)$ a trigonometric polynomial, there are no nonzero solutions to (1.8).

Remark 1.2 Although Theorem 1applies only to compactly supported initial conditions, we use it to prove Theorem 2. This is possible because compactly supported functions are a dense subset of $L^{2}(\mathbb{R})$; hence if we assumed ionization failed for some $\psi_{0}(x) \in L^{2}(\mathbb{R})$, we could approximate it by compactly supported functions and derive a contradiction.

Remark 1.3 If $\psi_{0}(x)$ is not compactly supported, then there can be other exponentially decaying components in (1.6). These come from the initial data being spread out over all space and are present even for solutions of the free Schrödinger equation. They also vary with $\psi_{0}(x)$, unlike $\psi_{b}(x, t)$. We assume that $\psi_{0}(x)$ is compactly supported simply to remove this technical point, not for any fundamental reason. See Remark 3.6 for an explanation how one might proceed with $\psi_{0}(x)$ not compactly supported.

\subsection{Equivalent formulations}

Here we describe some equivalent formulations of (1.1). This material is essentially taken from chapter 7 of [12]. We will use (1.13) in the proof of Theorem 2 and (1.12) in the proof of Theorem 1 We first define some auxiliary functions:

$$
\begin{gathered}
a(t)=\int_{0}^{t} b(s)^{2} d s \equiv a_{0} t+a_{v}(t) \\
b(t)=\sum_{n=1}^{\infty}\left(\frac{E_{n}}{i n \omega} e^{i n \omega t}+\frac{\bar{E}_{n}}{-i n \omega} e^{-i n \omega t}\right) \\
c(t)=2 \sum_{n=1}^{\infty}\left(\frac{E_{n}}{(i n \omega)^{2}} e^{i n \omega t}+\frac{\bar{E}_{n}}{(-i n \omega)^{2}} e^{-i n \omega t}\right) \equiv \sum_{n=1}^{\infty}\left(C_{n} e^{i n \omega t}+\bar{C}_{n} e^{-i n \omega t}\right)
\end{gathered}
$$

where $a_{v}(t)$ is $2 \pi / \omega$ periodic and has mean 0 , and $a_{0}=(\omega / 2 \pi) \int_{0}^{2 \pi / \omega} b(s)^{2} d s$. Note that $(1 / 2) c^{\prime \prime}(t)=b^{\prime}(t)=E(t)$.

Define $\psi_{v}(x, t) \equiv e^{+i a(t)} e^{+i b(t)(x-c(t))} \psi(x-c(t), t)$; then the following equation for $\psi_{v}$ is equivalent to (1.1):

$$
i \partial_{t} \psi_{v}(x, t)=\left(-\frac{\partial^{2}}{\partial x^{2}}-2 \delta(x-c(t))\right) \psi_{v}(x, t)
$$

This is the velocity gauge, and the equivalence can be verified by a computation ${ }^{2}$. Similarly, there is an equivalent equation in the magnetic gauge. We obtain it

\footnotetext{
${ }^{2}$ Equation 1.12] differs from what one finds in [12. In 12, they take $\tilde{b}(t)=\int_{0}^{t} E(s) d s$ and $\tilde{c}(t)=\int_{0}^{t} b(t) d t$, which implies that $\tilde{c}(t)=c(t)+c_{0}+c_{v} t$. Regardless, the essential feature, namely $(1 / 2) c^{\prime \prime}(t)=b^{\prime}(t)=E(t)$ is preserved.
} 
by setting $\psi_{B}(x, t)=e^{+i a(t)} e^{+i b(t) x} \psi(x, t)$ :

$$
i \partial_{t} \psi_{B}(x, t)=\left(-\frac{\partial^{2}}{\partial x^{2}}-2 \delta(x)+2 i b(t) \partial_{x}\right) \psi_{B}(x, t)
$$

Remark 1.4 Suppose that either $\psi_{B}(x, t)$ or $\psi_{v}(x, t)$ are time-periodic solutions of (1.13) or (1.12). Then $\psi(x, t)$ is a time quasi-periodic solution of (1.1), and $e^{i a_{0} t} \psi(x, t)$ is time-periodic.

\subsection{Organization of the paper}

In Section 2 we assume Theorem 10 to be true and use it to prove Theorem[2 In Section 3 we prove Theorem 1 In Section 4 we make some concluding remarks, and discuss possible directions of future research. Some technical material is presented in appendices.

\section{Ionization}

Assuming Theorem 10 to be true, we need to show that the Floquet equation (1.8) in the magnetic gauge has no nonzero solutions. This implies ionization for compactly supported initial data. Since compactly supported functions are a dense subset of $L^{2}(\mathbb{R})$, this implies ionization for all $\psi_{0}(x) \in L^{2}(\mathbb{R})$.

We prove that there are no solutions to (1.8) by taking a hypothetical solution $\psi_{b}(x, t)$ and showing it must be zero.

In Section 2.1 we solve (1.13) without a binding potential (the $-2 \delta(x)$ term) and characterize the solutions. We then expand the hypothetical bound state $\psi_{b}(x, t)$ in this basis, and derive necessary conditions on the coefficients to meet the boundary conditions (decay at $x= \pm \infty$ and continuity at $x=0$ ).

In Section 2.2 we use the characterization of solutions we constructed in Section 2.1 and show for $E(t)$ a trigonometric polynomial that it is impossible to construct solutions to (1.13) which are continuous at the origin. The basic technique is to analytically continue, in the $t$ variable, both $\psi_{B}\left(0_{-}, t\right)$ and $\psi_{B}\left(0_{+}, t\right)$ (which we suppose are equal) and use the Phragmen-Lindelof theorem to show that an associated function must be entire and bounded (and therefore constant). This implies that any solution to (1.8) must be zero, and ionization occurs.

\subsection{Solutions to the free problem}

By Theorem 10 we need to show that (1.8) has no nontrivial solutions. After switching to the magnetic gauge, we observe that this is the same as showing that if $\psi_{b}(x, t)$ solves

$$
\sigma_{b} \psi_{b}(x, t)=\left(-i \partial_{t}-\partial_{x}^{2}-2 \delta(x)+2 i b(t) \partial_{x}\right) \psi_{b}(x, t)
$$

with boundary conditions (1.8b) and (1.8c), then $\psi_{b}(x, t)=0$. 
We first study a simpler problem, namely (2.1) without the $\delta$-function binding potential.

$$
\sigma_{b} \psi(x, t)=\left(-i \partial_{t}-\partial_{x}^{2}+2 i b(t) \partial_{x}\right) \psi(x, t)
$$

Taking $\psi(x, t)=e^{\lambda x} \varphi_{\lambda}(t)$ as an ansatz, we obtain an ODE for $\varphi_{\lambda}(t)$ :

$$
\partial_{t} \varphi_{\lambda}(t)=-i\left(-\sigma-\lambda^{2}+2 i \lambda b(t)\right) \varphi_{\lambda}(t)
$$

This has the following family of solutions (recalling that $c^{\prime}(t)=2 b(t)$ ):

$$
\begin{gathered}
\varphi_{\lambda}(t)=e^{-i E_{\lambda} t} e^{\lambda c(t)} \\
E_{\lambda}=-\sigma-\lambda^{2}
\end{gathered}
$$

To ensure $2 \pi / \omega$ periodicity (in time), we must have $\left(-\sigma-\lambda^{2}\right)=m \omega, m \in \mathbb{Z}$. This implies that $\lambda= \pm i \sqrt{m \omega+\sigma}$ (the branch cut of $\sqrt{z}$ is taken to be $-i \mathbb{R}^{+}$). Therefore, (2.2) has the family of solutions:

$$
\begin{aligned}
\varphi_{m, \pm}(x, t) & =e^{ \pm \lambda_{m} x} e^{-i m \omega t} e^{ \pm \lambda_{m} c(t)} \\
\lambda_{m} & =-i \sqrt{\sigma+m \omega}
\end{aligned}
$$

\subsection{Matching solutions}

Given the family of solutions to (2.2), we can attempt to solve (1.13) assuming we know $\psi_{b}(0, t)$. We have four boundary conditions to satisfy (applying Theorem 1):

$$
\begin{gathered}
\psi_{b}(0, t)=\psi_{b}\left(0_{-}, t\right)=\psi_{b}\left(0_{+}, t\right) \\
\partial_{x} \psi_{b}\left(0_{+}, t\right)-\partial_{x} \psi_{b}\left(0_{-}, t\right)=-2 \psi_{b}(0, t) \\
\lim _{x \rightarrow \infty} \psi_{b}(-x, t)=\lim _{x \rightarrow \infty} \psi_{b}(+x, t)=0
\end{gathered}
$$

Consider now a hypothetical solution $\psi_{b}(x, t)$. We can expand $\psi(x, t)$ in terms of the functions $\varphi_{m, \pm}$ in the regions $x<0$ and $x>0$ separately (we neither need nor prove this fact, but all solutions of (2.2) can be expanded in this way). Thus we can expand $\psi_{b}(x, t)$ as follows:

$$
\psi_{b}(x, t)= \begin{cases}\sum_{m \in \mathbb{Z}}\left(\psi_{m,+}^{L} \varphi_{m,+}(x, t)+\psi_{m,-}^{L} \varphi_{m,-}(x, t)\right), & x \leq 0 \\ \sum_{m \in \mathbb{Z}}\left(\psi_{m,+}^{R} \varphi_{m,+}(x, t)+\psi_{m,+}^{R} \varphi_{m,+}(x, t)\right), & x \geq 0\end{cases}
$$

We now state our first result.

Proposition 2.1 Let $\psi_{b}(x, t)$ be a solution of (1.8) in the magnetic gauge. Then there exists a pair of sequences $\psi_{m, \pm} \in l^{2}(\mathbb{Z})$ such that:

$$
\psi_{b}(x, t)= \begin{cases}\sum_{m<1} \psi_{m}^{L} \varphi_{m,+}(x, t), & x \leq 0 \\ \sum_{m<1} \psi_{m}^{R} \varphi_{m,-}(x, t), & x \geq 0\end{cases}
$$

The equality holds pointwise. 
We only sketch a heuristic argument why the oscillating and growing components are absent; the proof can be found in Appendix A It is straightforward, but uses results proved in Section 3

For $m \geq 1$ (recalling $\sigma_{b} \in[0, \omega)$ and examining (2.5b) the functions $\varphi_{m, \pm}(x, t)$ are oscillatory in $x$ as $x \rightarrow \pm \infty$. Thus, if the coefficients $\psi_{m, \pm}^{L, R}(m \geq 1)$ were not zero, then $\psi_{b}(x, t)$ would not decay as $x \rightarrow \pm \infty$, violating (2.6c).

Similarly, we observe that $\varphi_{m,+}(x, t)$ are exponentially growing when $m<1$ as $x \rightarrow+\infty$, so $\psi_{m,+}^{R}$ must similarly be zero. The same argument applied to the region $x<0$ shows that $\psi_{m,+}^{L}$ must be zero when $m<1$. Therefore after dropping the \pm in the coefficients $\psi_{m, \pm}^{L, R}$, we obtain the result we seek.

Substituting (2.8) into the continuity condition (2.6a) yields:

$$
\sum_{m<1} \psi_{m}^{L} e^{-i m \omega t} e^{\lambda_{m} c(t)}=\sum_{m<1} \psi_{m}^{R} e^{-i m \omega t} e^{-\lambda_{m} c(t)}
$$

We now state a fact about the decay of the Fourier coefficients of the hypothetical Floquet solution. The proof of this fact uses the results of Section [3] and will be deferred to Appendix B

Proposition 2.2 Suppose $E(t)$ is a trigonometric polynomial with highest mode $N$, that is $E(t)=\sum_{n=1}^{N}\left(E_{n} e^{i n \omega t}+\bar{E}_{n} e^{-i n \omega t}\right)$. Set $z=e^{-i \omega t}$. Then $\psi_{b}(0, t)$ has the decomposition:

$$
\psi_{b}(0, t)=f(z)+g\left(z^{-1}\right)
$$

The functions $f(\cdot)$ and $g(\cdot)$ are entire functions of exponential order $2 N$, and $g(0)=0$. This shows in particular that $\psi_{b}(0, t)$ is continuous.

The correspondence between $\psi_{b}(0, t)$ and $f(z), g(z)$ is as follows. Let $\psi_{j}$ denote the $j$ 'th Fourier coefficient of $\psi_{b}(0, t)$, that is $\psi_{b}(0, t)=\sum_{j} \psi_{j} e^{i j \omega t}$. Then letting $f_{j}, g_{j}$ be the Taylor coefficients of $f(z), g(z)$, we find $f_{j}=\psi_{-j}$ for $j \geq 0$ and $g_{j}=\psi_{j}$ for $j<0$.

Finally, we state a result we use, proved in most complex analysis textbooks, e.g. [29].

Theorem 3 (Phragmen-Lindelof) Let $f(z)$ be of exponential order $2 N$, that is $|f(z)| \leq C e^{C^{\prime}|z|^{2 N}}$. Let $S$ be a sector of opening smaller than $\pi / 2 N$. Then:

$$
\sup _{z \in \partial S}|f(z)| \geq \sup _{z \in S}|f(z)|
$$

We are now prepared to prove the main result.

Proof of Theorem 2 . The basic idea of the proof is as follows. We assume the existence of a $\psi_{b}(x, t)$ satisfying (2.8). We then study the behavior of $\psi_{b}(0, t)$ in the complex $t$ plane and show $\psi_{b}(0, t)$ must be zero.

We describe the case $N=1$ now (i.e. $E(t)=E \cos (\omega t)$; the case $N \neq 2$ is treated below). The key idea is that we can use (2.8) to obtain an asymptotic expansion of $\psi_{b}\left(0_{+}, t\right)$ and $\psi_{b}\left(0_{-}, t\right)$ on the open right and left half planes in the variable $z=e^{-i \omega t}$ (respectively); to leading order $\psi_{b}\left(0_{-}, t\right) \sim \psi_{m}^{L} z^{m} e^{-C|\Re z|}$ 
and $\psi_{b}\left(0_{+}, t\right) \sim \psi_{m}^{R} z^{m} e^{-C|\Re z|}$ (note that $m$ and $C$ may be different). This asymptotic expansion shows that $f(z)$ decays exponentially along any ray $z=$ $r e^{i \phi}$ in the open left or right half planes.

In fact, the asymptotic expansion allows us to observe that $f(z)$ (the part of $\psi_{b}(0, t)$ which is analytic in $\left.z\right)$ must be bounded except possibly on the line $i \mathbb{R}$. The Phragmen-Lindelof Theorem (Theorem 31) combined with Proposition 2.2 allows us to conclude that $f(z)$ is bounded on the line $i \mathbb{R}$. This shows $f(z)$ is bounded on $\mathbb{C}$ and hence zero.

Since $f(z)$ is zero, $\psi_{b}(0, t)=g(z) \sim g_{M} z^{-M}$ for some $M \in \mathbb{N}$ (since $g(z)$ is analytic). But we previously said also that $\psi_{b}(0, t) \sim \psi_{m}^{L} z^{m} e^{-C|\Re z|}$. Two asymptotic expansions must agree to leading order; the only way this can happen is if $g(z)=\psi_{b}(0, t)=0$.

The main difference between the case $N=1$ (monochromatic field) and $N>$ 1 (polychromatic field) is that instead of the exponential asymptotic expansions being valid on the left and right half planes, they are valid on sectors of opening $\pi / N$; this we need to apply Theorem 3 to the boundaries of these sectors.

We now go through the details.

Step 1: Setup

By Theorem[1] we need to show that (1.8) has no nonzero solutions. Toward that end, let $\psi_{b}(x, t)$ be a hypothetical solution to (1.8). By the hypothesis of Theorem 2 we let $E(t)$ be a nonzero trigonometric polynomial of order $N$.

Let $z=e^{-i \omega t}$. Let $\mathfrak{C}(z)=\sum_{j=1}^{N}\left(\bar{C}_{j} z^{j}+C_{j} z^{-j}\right)$ where the $C_{j}$ are the coefficients from (1.11c). We apply Proposition 2.2 to $\psi_{b}(0, t)$ and (2.9) to obtain:

$$
\begin{aligned}
\psi_{b}(0, t)=f(z)+g\left(z^{-1}\right) & \\
=\sum_{m<1} \psi_{m}^{L} z^{m} e^{+\lambda_{m} \mathfrak{C}(z)} & =\sum_{m<1} \psi_{m}^{R} z^{m} e^{-\lambda_{m} \mathfrak{C}(z)}
\end{aligned}
$$

The first equality holds by (2.10), the second by (2.8) with $x=0$. A priori, equality holds only when $|z|=1$. However, either of the latter two sums are analytic in any neighborhood in which they are uniformly convergent. Thus, $f(z)+g\left(z^{-1}\right)$ is the analytic continuation of the sum if the sum is convergent on any neighborhood containing part of the unit disk.

For the rest of this proof, we make the following convention. The functions $\psi^{L, R}(z)$ are defined by

$$
\begin{aligned}
& \psi^{L}(z)=\sum_{m<1} \psi_{m}^{L} z^{m} e^{+\lambda_{m} \mathfrak{C}(z)} \\
& \psi^{R}(z)=\sum_{m<1} \psi_{m}^{R} z^{m} e^{-\lambda_{m} \mathfrak{C}(z)}
\end{aligned}
$$

for those $z$ where the sum is convergent.

Step 2: Convergence of the sum

We show now that the sum in (2.11) is convergent on a sufficiently large region. 
For $|z| \geq 1$ and $\Re \mathfrak{C}(z)>0$, consider the sum $\sum_{m<1} \psi_{m}^{R} z^{m} e^{-\lambda_{m} \mathfrak{C}(z)}$. In this region, since $\Re \mathfrak{C}(z)>0$, we find that $e^{\lambda_{m} \mathfrak{C}(z)} \leq 1$. The coefficients $\psi_{m}^{L, R}$ are bounded uniformly in $m$ (since they are an $l^{2}$ sequence, by Proposition 2.1). For $|z|>1, z^{m}$ is geometrically decaying as $m \rightarrow-\infty$. Therefore the series is absolutely convergent when $|z|>1$ and $\Re \mathfrak{C}(z)>0$.

The same statement holds with $\sum_{m<1} \psi_{m}^{L} z^{m} e^{+\lambda_{m} \mathfrak{C}(z)}$ in the region where $\Re \mathfrak{C}(z)<0$.

Let us define the following sets:

$$
\begin{array}{r}
S^{+}=\{z \in \mathbb{C}:|z| \geq 1, \Re \mathfrak{C}(z)>0 \text { and also } z \text { is connected in } \\
\{z: \Re \mathfrak{C}(z)>0\} \text { to the unit circle }\} \\
S^{-}=\{z \in \mathbb{C}:|z| \geq 1, \Re \mathfrak{C}(z)<0 \text { and also } z \text { is connected in } \\
\{z: \Re \mathfrak{C}(z)<0\} \text { to the unit circle }\}
\end{array}
$$

A schematic diagram indicating the structure of these sectors is shown in Figure 1 for the case where $N=2$.

By Proposition 2.2 we see that $\psi^{R}(z)$ is analytic in $S^{+}$and $\psi^{L}(z)$ is analytic in $S^{-}$, since the sum in (2.12) is convergent there.

We now show that all connected components of $S^{+}$and $S^{-}$must be unbounded if $\mathfrak{C}(z)$ is non constant. Suppose either $S^{+}$or $S^{-}$had a bounded connected component, denoted by $B$. Then $\Re \mathfrak{C}(z)$ would be zero on $\partial B$. By the real max modulus principle, $\Re \mathfrak{C}(z)$ would be zero inside $B$, and hence it would be zero everywhere.

Finally we show that the regions $S^{+}$and $S^{-}$"fill out" to open sectors as $|z| \rightarrow \infty$. That is to say, if $S$ is some sector in which $\Re z^{N}>0$, then for any ray $\left\{r e^{i \theta}: r>1\right\}$ contained in $S, \exists R=R(\theta)$ so that the truncated ray $\left\{r e^{i \theta}: r>R(\theta)\right\} \subset S^{+}$.

Without loss of generality ${ }^{3}$, let us suppose that $C_{N} \in \mathbb{R}^{+}$. For very large $|z|$, we write $\mathfrak{C}(z)=\sum_{j=1}^{N} \bar{C}_{j} z^{j}+C_{j} z^{-j}=\bar{C}_{n} z^{N}+O\left(z^{N-1}\right)$. Then setting $z=r e^{i \theta}$, we find that $r^{-N} \mathfrak{C}\left(r e^{i \theta}\right)=\bar{C}_{N} e^{i N \theta}+O\left(r^{-1}\right)$. Thus, for $r$ sufficiently large and $N \theta \neq(2 m+1) \pi / 2$, we find that $r^{-N} \mathfrak{C}\left(r e^{i \theta}\right)$ has either strictly positive real part or strictly negative real part. In particular, if $|N \theta \mp \pi / 2|>\epsilon$, then $\exists R=R(\epsilon, \theta)$ so that $\Re r^{-N} \mathfrak{C}\left(r e^{i \theta}\right)$ is bounded strictly away from zero.

Motivated by the above, we define the following subsets of $\mathbb{C}$ (with $j=$ $0 \ldots N-1)$ :

$$
\begin{aligned}
& A_{j, \epsilon}^{+}=\left\{r e^{i \theta}: r \geq R(\epsilon, \theta),\right. \\
& \quad \theta \in[-\pi j / 2 N+2 \pi j / N+\epsilon, \pi / 2 N+2 \pi j / N-\epsilon]\} \\
& A_{j, \epsilon}^{-}=\left\{r e^{i \theta}: r \geq R(\epsilon, \theta),\right. \\
& \quad \theta \in[-\pi / 2 N+2 \pi(j+1 / 2) / N+\epsilon, \pi / 2 N+2 \pi(j+1 / 2) / N-\epsilon]\}
\end{aligned}
$$

\footnotetext{
${ }^{3}$ Suppose $C_{N}=\rho e^{i \theta}$. Then rather than choosing $z=e^{i \omega t}$, we would substitute $z=$ $e^{i(\omega t-\theta / N)}$.
} 
Clearly, for sufficiently large $R, A_{j, \epsilon}^{+} \backslash B_{R} \subset S^{+}$and $A_{j, \epsilon}^{-} \backslash B_{R} \subset S^{-}$.

Step 3: Asymptotics of $f(z)$

We now show $f(z)=0$. We begin by writing $f(z)$ as follows:

$$
\begin{aligned}
& f(z)=\sum_{n=0}^{\infty} f_{n} z^{n}=-\sum_{n=1}^{\infty} g_{n} z^{-n}+\sum_{m<1} \psi_{m}^{R} z^{m} e^{-\lambda_{m} \mathfrak{C}(z)}, z \in S^{+} \\
& f(z)=\sum_{n=0}^{\infty} f_{n} z^{n}=-\sum_{n=1}^{\infty} g_{n} z^{-n}+\sum_{m<1} \psi_{m}^{L} z^{m} e^{+\lambda_{m} \mathfrak{C}(z)}, z \in S^{-}
\end{aligned}
$$

We let the sectors $S_{k}, k=0 \ldots 2 N+1$ be a set of sectors of opening $\pi /(2 N+1)$ arranged in such a way that the boundaries of $S_{k}$ avoid the rays $r e^{i \pi(2 j+1) / 2 N}$. Therefore, for sufficiently large $|z|$, the boundaries of $S_{k}$ are contained in either the region $A_{j, \epsilon}^{+}$or $A_{j, \epsilon}^{-}$except for a compact region. On $\partial S_{k}, f(z)$ is decaying as $|z| \rightarrow \infty$, by a simple examination of (2.14). For $|z|$ small, we observe that $f(z)$ is entire, and therefore bounded on compact regions.

We have shown that $f(z)$ is bounded on $\partial S_{k}$. Applying the PhragmenLindelof theorem, $f(z)$ is therefore bounded on $S_{k}$. Since $\cup_{k=0}^{2 n+1} S_{k}=\mathbb{C}$, we find $f(z)$ is constant. Since we know that along any ray contained in $A_{j, \varepsilon}^{ \pm}, f(z)$ is decreasing, we know $f(z)=0$.

Step 4: Asymptotics of $g(z)$

We will now show that $g(z)=0$. We rewrite (2.14) with $g(z)$ on the left side.

$$
\begin{aligned}
& \sum_{n=1}^{\infty} g_{n} z^{-n}=\sum_{m<1} \psi_{m}^{R} z^{m} e^{-\lambda_{m} \mathfrak{C}(z)}, z \in S^{+} \\
& \sum_{n=1}^{\infty} g_{n} z^{-n}=\sum_{m<1} \psi_{m}^{L} z^{m} e^{+\lambda_{m} \mathfrak{C}(z)}, z \in S^{-}
\end{aligned}
$$

Since the left sides of (2.15a) and (2.15b) are (convergent) asymptotic power series (for sufficiently large $|z|$ ), while the right sides of (2.15a) and (2.15b) are (convergent) asymptotic series of exponentials, we find that the right side decays much faster than the left side. This is impossible unless both sides are zero.

To make this clearer, we can rewrite 2.15a) as (with 2.15b treated similarly):

$$
g_{j} z^{-j}(1+o(1))=\psi_{k}^{R} z^{k} e^{-\lambda_{k} \mathfrak{C}(z)}(1+o(1))
$$

We can rewrite this as:

$$
g_{j}^{-1} \psi_{k}^{R} z^{k+j} e^{-\lambda_{k} \mathfrak{C}(z)}=\frac{(1+o(1))}{(1+o(1))}
$$

Here, $j$ is the smallest integer so that $g_{j} \neq 0$ and $k$ is the smallest integer so that $\psi_{k}^{R} \neq 0$.

The limit of the left side is 0 as $|z| \rightarrow \infty$ (inside the region $A_{j, \epsilon}^{+}$). But the limit of the right side is 1 . This is impossible. 


\section{The Floquet Formulation}

In this section we prove Theorem 1 This is done by studying the time dependent solution of (1.1). To do so we define an auxiliary function $Y(t)=\psi(c(t), t)$ and derive a closed integral equation (of Volterra type) for it via Duhamel's formula.

We then apply the Zak transform (defined below) in time to the integral equation for $Y(t)$. This yields an integral equation of compact Fredholm type for $\mathcal{Z}[f](\sigma, t)$, the Zak transform of $Y(t)$. The integral operator is shown to be analytic in $\sigma$. The analytic Fredholm alternative to this equation shows that $\mathcal{Z}[f](\sigma, t)$ is meromorphic in $\sigma^{1 / 2}$ with one pole. The pole corresponds to a resonance or bound state, while the branch point corresponds to the dispersive part of the solution.

In Section 3.3 we extend these results from $x=0$ to the entire real line. We show that the wavefunction, considered in the magnetic gauge, can be decomposed in the form (1.6). If $\Im \sigma_{b}=0$, then $\Re \sigma_{b} \in(0, \omega)$ and $\psi_{b}(x, t)$ corresponds to a Floquet bound state. The wavefunction $\psi_{d}(x, t)$ decays with time, in particular $\left\|\psi_{d}(x, t)\right\|_{L^{\infty}} \leq C\langle t\rangle^{-1 / 2}$, where $\langle t\rangle=\left(1+t^{2}\right)^{1 / 2}$.

\subsection{Setting up the problem}

Here we work in the velocity gauge and study (1.12). We rewrite (1.1) in Duhamel form, using the standard Green's function for the free Schrödinger equation:

$$
\begin{aligned}
& \psi_{v}(x, t)=\psi_{v, 0}(x, t) \\
& \quad+2 i \int_{0}^{t} \int_{\mathbb{R}} \exp \left(\frac{i\left(x-x^{\prime}\right)^{2}}{4\left(t-t^{\prime}\right)}\right) \delta\left(x^{\prime}-c\left(t^{\prime}\right)\right) \psi_{v}\left(x^{\prime}, t^{\prime}\right) d x^{\prime} \frac{d t^{\prime}}{\sqrt{4 \pi i\left(t-t^{\prime}\right)}}
\end{aligned}
$$

where we have defined:

$$
\psi_{v, 0}(x, t)=e^{i \partial_{x}^{2} t} \psi_{v}(x, 0)
$$

If we do the $x^{\prime}$ integral explicitly and change variables to $s=t-t^{\prime}$, we find:

$$
\begin{aligned}
\psi_{v}(x, t)=\psi_{v, 0}(x, t) & \\
& +2 i \int_{0}^{t} \exp \left(\frac{i(x-c(t-s))^{2}}{4 s}\right) \psi_{v}(c(t-s), t-s) \frac{d s}{\sqrt{4 \pi i s}}
\end{aligned}
$$

We now substitute $x=c(t)$, to obtain a closed equation for $\psi_{v}(c(t), t)$ :

$$
\begin{aligned}
\psi_{v}(c(t), t)= & \psi_{v, 0}(c(t), t) \\
& +\sqrt{\frac{i}{\pi}} \int_{0}^{t} \exp \left(\frac{i(c(t)-c(t-s))^{2}}{4 s}\right) \psi_{v}(c(t-s), t-s) \frac{d s}{\sqrt{s}}
\end{aligned}
$$

Set $Y_{0}=\psi_{v, 0}(c(t), t)$ and $Y(t)=\psi(c(t), t)$ to obtain:

$$
Y(t)=Y_{0}(t)+\sqrt{\frac{i}{\pi}} \int_{0}^{t} \exp \left(\frac{i(c(t)-c(t-s))^{2}}{4 s}\right) Y(t-s) \frac{d s}{\sqrt{s}}
$$


We will show that either $Y(t) \rightarrow 0$ as $t \rightarrow \infty$ or (3.4) has quasi-periodic in time solutions. The main tool of our analysis will be the Zak transform.

Definition 3.1 Let $f(t)=0$ for $t<0$ and $|f(t)| \leq C e^{\alpha t}\left(\alpha \in \mathbb{R}^{+}\right)$. Then $f(t)$ is said to be Zak transformable. The Zak transform of $f(t)$ is defined (for $\Im \sigma>\alpha)$ by:

$$
\mathcal{Z}[f](\sigma, t)=\sum_{j \in \mathbb{Z}} e^{i \sigma(t+2 \pi j / \omega)} f(t+2 \pi j / \omega)
$$

and by the analytic continuation of (3.5) when $\Im \sigma<\alpha$. $\mathcal{Z}[f](\sigma, t)$ has the following properties:

$$
\begin{gathered}
f(t)=\omega^{-1} \int_{i \alpha}^{i \alpha+\omega} e^{-i \sigma t} \mathcal{Z}[f](\sigma, t) d \sigma \\
\mathcal{Z}[f](\sigma, t+2 \pi / \omega)=\mathcal{Z}[f](\sigma, t) \\
\mathcal{Z}[f](\sigma+\omega, t)=e^{i \omega t} \mathcal{Z}[f](\sigma, t) \\
\mathcal{Z}[p f](\sigma, t)=p(t) \mathcal{Z}[f](\sigma, t)
\end{gathered}
$$

In (3.6d), $p(t)$ is $2 \pi / \omega$ periodic.

Remark 3.2 Suppose $f(t)$ is Zak transformable, and uniformly bounded in time $(\alpha=0)$. Suppose further that the analytic continuation of $\mathcal{Z}[f](\sigma, t)$ has a singularity (say at $\sigma=0$ ). Then (3.6c) still holds, in the sense that for any direction $\theta, \mathcal{Z}[f]\left(\sigma+\omega+0 e^{i \theta}, t\right)=e^{i \omega t} \mathcal{Z}[f]\left(\sigma+0 e^{i \theta}, t\right)$.

Remark 3.3 More information on the Zak transform can be found in, e.g., [13, p.p. 109-110]. Our definition differs slightly from that in [13] - we let $\sigma$ take complex values.

Remark 3.4 One can relate the Zak and Fourier transforms as follows. Let $\hat{f}=\int e^{-i k t} f(t) d t$ denote the Fourier transform of $f(t)$. Then:

$$
\mathcal{Z}[f](\sigma, t)=\frac{\omega}{2 \pi} \sum_{n \in \mathbb{Z}} \hat{f}(\sigma+n \omega) e^{-i n \omega t}
$$

This follows by applying the Poisson sum formula to (3.5). In fact, (3.6a) can be derived immediately from this relation.

This relation implies that our approach is equivalent to the Fourier/Laplace transform analysis done in 11, 8, 9]. The Zak transform is used simply for algebraic convenience.

We proceed as follows. Applying the Zak transform to (3.4) yields an integral equation of the form

$$
y(\sigma, t)=y_{0}(\sigma, t)+K(\sigma) y(\sigma, t)
$$


with $y(\sigma, t)=\mathcal{Z}[Y](\sigma, t), y_{0}(\sigma, t)=\mathcal{Z}\left[Y_{0}\right](\sigma, t)$ and $K(\sigma)$ the Zak transform of the integral operator in (3.4). $K(\sigma)$ will be shown to be meromorphic in $\sigma$ as a compact operator family from $L^{2}\left(S^{1}, d t\right) \rightarrow L^{2}\left(S^{1}, d t\right)$, except for a branch point at $\sigma=0$.

We will then use the Fredholm alternative theorem to invert $(1-K(\sigma))$. Once this is done, we find:

$$
y(\sigma, t)=(1-K(\sigma, t))^{-1} y_{0}(\sigma, t)
$$

The poles of $(1-K(\sigma))^{-1}$ will correspond to resonances, and a branch point at $\sigma=0$ will correspond to the dispersive part of the solution.

To begin, we determine the analyticity properties of $\mathcal{Z}\left[Y_{0}\right](\sigma, t)$.

Proposition 3.5 Suppose $\psi_{0}(x)$ is smooth and compactly supported. Then near $\sigma=0, y_{0}(\sigma, t)$ has the expansion:

$$
\mathcal{Z}\left[Y_{0}\right](\sigma, t)=y_{0}(\sigma, t)=\sigma^{-1 / 2}(1 / 2) \int_{\mathbb{R}} \psi_{0}(x) d x+f\left(\sigma^{1 / 2}, t\right)
$$

The function $f\left(\sigma^{1 / 2}, t\right)$ is analytic in $\sigma^{1 / 2}$, and is in $L^{2}\left(S^{1}, d t\right)$. Also:

$$
\left\|\mathcal{Z}\left[Y_{0}\right](\sigma, t)\right\|_{L^{2}\left(S^{1}, d t\right)} \leq C_{1} e^{C_{2}|\Im \sigma|}
$$

Proof. Consider $Y_{0}(t)=\psi_{v, 0}(c(t), t)$ for $t \geq 0$ only (and $Y_{0}(t)=0$ for $t<0$ ). Then (with slight abuse of notation):

$$
Y_{0}(t)=\chi_{\mathbb{R}^{+}}(t) \int_{\mathbb{R}} e^{i k c(t)} e^{i k^{2} t} \hat{\psi}_{0}(k) d k
$$

Computing the Zak transform yields:

$$
\begin{gathered}
\mathcal{Z}\left[Y_{0}\right](\sigma, t)=\sum_{j \in \mathbb{Z}} e^{i \sigma(t-2 \pi j / \omega)} \chi_{\mathbb{R}^{+}}(t-2 \pi j / \omega) \int_{\mathbb{R}} e^{i k c(t)} e^{i k^{2}(t-2 \pi j / \omega)} \hat{\psi}_{0}(k) d k \\
=\int_{\mathbb{R}} e^{i k c(t)} \hat{\psi}_{0}(k)\left[\sum_{j \in \mathbb{Z}} e^{i \sigma(t-2 \pi j / \omega)} e^{i k^{2}(t-2 \pi j / \omega)} \chi_{\mathbb{R}^{+}}(t-2 \pi j / \omega)\right] d k \\
=\int_{\mathbb{R}} e^{i k c(t)} \hat{\psi}_{0}(k)\left[\sum_{n \in \mathbb{Z}} \frac{e^{-i n \omega t}}{i\left(k^{2}+\sigma+n \omega\right)}\right] d k \\
=\sigma^{-1 / 2}(1 / 2) \int_{\mathbb{R}} \psi_{0}(y) d y+\sigma^{-1 / 2}(1 / 2) \int_{\mathbb{R}}\left(e^{-\sqrt{\sigma}|c(t)-y|}-1\right) \psi_{0}(y) d y \\
+\sum_{n \neq 0} \frac{e^{-i n \omega t}}{2 \sqrt{\sigma+n \omega}} \int_{\mathbb{R}} e^{-\sqrt{\sigma+n \omega}|c(t)-y|} \psi_{0}(y) d y
\end{gathered}
$$

The interchange of the sum and integral between lines 1 and 2 is justified (for $\Im \sigma>0$ and $t$ fixed) since the sum over $j$ is absolutely convergent, as is the integral over $k$. Th result is valid for arbitrary $\sigma$ by analytic continuation. 
The change inside the square brackets between lines 2 and 3 comes from the Poisson summation formula in the $t$ variable, and the fact that the Fourier transform of $\chi_{\mathbb{R}^{+}}(t) e^{i\left(k^{2}+\sigma\right) t}$ is $-i\left(k^{2}+\sigma+\zeta\right)^{-1}$ (with $\zeta$ dual to $t$ ).

The first term on the right side of (3.10) agrees with that in (3.9). Since $\left(e^{-\sqrt{\sigma}|c(t)-y|}-1\right)$ is analytic in $\sigma^{1 / 2}$, the second term is analytic in $\sigma^{1 / 2}$. The second and third (which is analytic in $\sigma$ ) terms become $f(\sigma, t)$.

Since $\psi_{0}(x)$ is supported on a compact region, $|c(t)-y|$ is bounded (say by $C_{2}$ ) and exponential growth follows.

Remark 3.6 Suppose that instead of being compactly supported, $\psi_{0}(x)=$ $e^{-|x|}$, the bound state of $-\Delta-2 \delta(x)$. In that event $\hat{\psi}_{0}(k)=1 /\left(k^{2}+1\right)$ which is singular at $k= \pm i$. We can carry through the same computation, but $\mathcal{Z}\left[Y_{0}\right](\sigma, t)$ will have an extra pole at $\Im \sigma=-1$ stemming from the poles of $\hat{\psi}_{0}(k)$. This pole corresponds to an exponentially decaying component of $e^{i \Delta t} \psi_{0}(x)$. When $Y(t)$ and $\psi(x, t)$ are reconstructed, this pole will correspond to a similar exponentially decaying component of $\psi_{d}(x, t)$. This pole is not a resonance; a resonance is a pole which is created by the potential and which is not present under the free flow.

We now determine the Zak transform of the integral operator in (3.4) and it's resolvent.

\subsection{Construction of the resolvent}

We now apply the Zak transform to (3.4) to construct an equivalent integral equation.

Proposition 3.7 Let $f(t)$ be Zak transformable. Consider the integral operator:

$$
K_{V} f(t)=\sqrt{\frac{i}{\pi}} \int_{0}^{t} \exp \left(i \frac{(c(t)-c(t-s))^{2}}{s}\right) f(t-s) \frac{d s}{\sqrt{s}}
$$

Then if $\varsigma \sigma>0$, we find:

$$
\begin{aligned}
\mathcal{Z}\left[K_{V} f\right](\sigma, t) & =K(\sigma) f(\sigma, t) \\
= & \sqrt{\frac{i}{\pi}} \int_{0}^{\infty} \exp \left(i \frac{(c(t)-c(t-s))^{2}}{s}\right) e^{i \sigma s} \mathcal{Z}[f](\sigma, t-s) \frac{d s}{\sqrt{s}}
\end{aligned}
$$

Proof. Rewrite (3.11) as:

$$
\begin{aligned}
\sqrt{\frac{i}{\pi}} \int_{0}^{t} \exp \left(i \frac{(c(t)-c(t-s))^{2}}{s}\right) f(t-s) \frac{d s}{\sqrt{s}} \\
\quad=\sqrt{\frac{i}{\pi}} \int_{\mathbb{R}} \exp \left(i \frac{(c(t)-c(t-s))^{2}}{s}\right) f(t-s) \chi_{\mathbb{R}^{+}}(s) \frac{d s}{\sqrt{s}}
\end{aligned}
$$


Applying $\mathcal{Z}$ to both sides of 3.13 we get

$$
\begin{aligned}
& \mathcal{Z}\left[K_{V} f\right](\sigma, t)=\sum_{j \in \mathbb{Z}} e^{i \sigma(t+2 \pi j / \omega)}\left[K_{V} f\right](t) \\
&=\sqrt{\frac{i}{\pi}} \sum_{j \in \mathbb{Z}} e^{i \sigma(t+2 \pi j / \omega)} \int_{\mathbb{R}} \exp \left(i \frac{(c(t)-c(t-s))^{2}}{s}\right) f(t+2 \pi j / \omega-s) \chi_{\mathbb{R}^{+}}(s) \frac{d s}{\sqrt{s}} \\
&=\sqrt{\frac{i}{\pi}} \int_{\mathbb{R}} \exp \left(i \frac{(c(t)-c(t-s))^{2}}{s}\right) e^{i \sigma s} \\
& \times\left[\sum_{j \in \mathbb{Z}} e^{i \sigma(t-s+2 \pi j / \omega)} f(t-s+2 \pi j / \omega)\right] \chi_{\mathbb{R}^{+}}(s) \frac{d s}{\sqrt{s}} \\
&=\sqrt{\frac{i}{\pi}} \int_{0}^{\infty} \exp \left(i \frac{(c(t)-c(t-s))^{2}}{s}\right) e^{i \sigma s} \mathcal{Z}[f](\sigma, t-s) \frac{d s}{\sqrt{s}}
\end{aligned}
$$

This is what we wanted to show.

We now show that the operator $K(\sigma)$, constructed above, is compact. We decompose $K(\sigma)$ as $K_{F}(\sigma)+K_{L}(\sigma)$ (defined shortly), and treat each piece separately.

Proposition 3.8 Define $K_{F}(\sigma): L^{2}\left(S^{1}, d t\right) \rightarrow L^{2}\left(S^{1}, d t\right)$ by:

$$
K_{F}(\sigma) f(t)=\sqrt{\frac{i}{\pi}} \int_{0}^{\infty} e^{i \sigma t} f(t-s) \frac{d s}{\sqrt{s}}
$$

Then $K_{F}(\sigma)$ is compact and analytic for $\Im \sigma \geq 0, \sigma \neq 0$. It has a $\sigma^{-1 / 2}$ branch point at $\sigma=0$, and can be analytically continued to $\Im \sigma<0$.

Proof. We compute this exactly by expanding $f(t)$ in Fourier series and interchanging the order of summation and integration:

$$
\sqrt{\frac{i}{\pi}} \sum_{n \in \mathbb{Z}} f_{n} e^{-i n \omega t} \int_{0}^{\infty} e^{i(\sigma+n \omega) s} \frac{d s}{\sqrt{s}}=\sum_{n \in \mathbb{Z}} \frac{f_{n}}{\sqrt{\sigma+n \omega}} e^{-i n \omega t}
$$

This is valid for $\Im \sigma>0$, as well as $\Im \sigma=0$ but in this case we must treat the integral as improper.

Thus, in the basis $e^{-i n \omega t}$, this operator is diagonal multiplication by $(\sigma+$ $n \omega)^{-1 / 2}$. Compactness follows since the diagonal elements decay in both directions. Analyticity for $\sigma \neq 0$ follows by inspection of the right side of (3.15), and choosing the branch cut of $\sqrt{\sigma+n \omega}$ to lie on the negative real line.

Proposition 3.9 Define $K_{L}(\sigma): L^{2}\left(S^{1}, d t\right) \rightarrow L^{2}\left(S^{1}, d t\right)$ as:

$$
K_{L}(\sigma) f(t)=\sqrt{\frac{i}{\pi}} \int_{0}^{\infty}\left[\exp \left(i \frac{(c(t)-c(t-s))^{2}}{s}\right)-1\right] e^{i \sigma s} f(t-s) \frac{d s}{\sqrt{s}}
$$


Then $K_{L}(\sigma)$ is compact for $\Im \sigma \geq 0$ and analytic for $\Im \sigma>0$. It has continuous limiting values at $\Im \sigma=0$. Near $\sigma=0, K_{L}(\sigma)$ is analytic in $\sigma^{1 / 2}$ and behaves to leading order like $O\left(\sigma^{1 / 2}\right)$.

Proof. Rewrite (3.16) as:

$$
\int_{0}^{2 \pi / \omega} \sum_{k=0}^{\infty}\left[\exp \left(i \frac{(c(t)-c(t-s))^{2}}{s+2 \pi k / \omega}\right)-1\right] \frac{e^{i \sigma(s+2 \pi k / \omega)}}{\sqrt{s+2 \pi k / \omega}} f(t-s) d s
$$

Provided $\Im \sigma \geq 0$, the sum is decaying at least as fast as $k^{-3 / 2}$. Each term in the sum is continuous. Thus the sum is absolutely convergent to a smooth function in $t$ and $s$. The region of integration is compact, and so is $K_{L}(\sigma)$.

To show the analyticity in $\sigma^{1 / 2}$, we change variables in (3.16) to $z=\sigma s$. Then (3.16) becomes:

$$
\sqrt{\frac{i}{\pi}} \int_{0}^{\infty e^{i P H \sigma}}\left[\exp \left(i \sigma \frac{(c(t)-c(t-z / \sigma))^{2}}{z}\right)-1\right] e^{i z} f(t-z / \sigma) \frac{d z}{\sqrt{\sigma z}}
$$

The integrand is analytic in $\sigma^{1 / 2}$ with no constant term, hence $K(\sigma)$ is analytic in $\sigma^{1 / 2}$ with leading order behavior $O\left(\sigma^{1 / 2}\right)$.

We now analytically continue $K_{L}(\sigma)$ to the strip $0 \leq \Re \sigma \leq \omega$.

Proposition 3.10 Let $K^{\prime}(\sigma)$ be the integral operator defined by:

$$
\begin{gathered}
K^{\prime}(\sigma) f(t)=\int_{0}^{2 \pi / \omega} k^{\prime}(t, s) f(t-s) d s \\
k^{\prime}(t, s)=\frac{\omega}{2 \pi i} \int_{\mathbb{R}+0 i} \frac{e^{\sigma p}}{1-e^{\omega p-i \omega s}}\left[\exp \left(\frac{(c(t)-c(t-s))^{2}}{p}\right)-1\right] \frac{d p}{\sqrt{p}}
\end{gathered}
$$

Then $K^{\prime}(\sigma)$ is analytic and compact for $0<\Re \sigma<\omega$, and vanishes as $\Im \sigma \rightarrow$ $\pm \infty$. In addition, $K^{\prime}(\sigma)$ is the analytic continuation of $K(\sigma)$.

\section{Proof.}

Step 1: Analyticity

For $\Re \sigma \in(0, \omega)$, we observe that the integrand in (3.18b) is exponentially decaying at $\pm \infty$. The integrand is singular only when $p=0$ and when $p=$ $s+2 \pi n$ for $n \in \mathbb{Z}$.

To show that the integral in (3.18b makes sense, we need to show it is finite. We do this by shifting the contour of integration. Let $\gamma(t)=t$ for $t \in \mathbb{R} \backslash[-2 \pi / \omega, 2 \pi / \omega]$, and $\gamma(t)=e^{i[\pi-(\omega t+2 \pi) / 4]}$ for $t \in[-2 \pi / \omega, 2 \pi / \omega]$. That is, $\gamma(t)$ travels along the real line, and circles upward around the disk of radius 
$2 \pi / \omega$. Then:

$$
\begin{aligned}
& \omega^{-1} k^{\prime}(t, s)= \int_{\mathbb{R}+0 i} \frac{e^{\sigma p}}{1-e^{\omega p-i \omega s}}\left[\exp \left(\frac{(c(t)-c(t-s))^{2}}{p}\right)-1\right] \frac{d p}{\sqrt{p}} \\
&= \int_{\gamma} \frac{e^{\sigma p}}{1-e^{\omega p-i \omega s}}\left[\exp \left(\frac{(c(t)-c(t-s))^{2}}{p}\right)-1\right] \frac{d p}{\sqrt{p}} \\
&+\frac{2 \pi i}{\omega} e^{i \sigma s}\left[\exp \left(\frac{(c(t)-c(t-s))^{2}}{i s}\right)-1\right] \frac{1}{\sqrt{i s}}
\end{aligned}
$$

The integrand in the first term is analytic since $p$ stays away from 0 (thus avoiding the essential singularity at $p=0$ ). It is exponentially decaying both for large positive $p$ (at the rate $e^{(\sigma-\omega) p}$ ) and for large negative $p$ (at the rate $\left.e^{-\sigma p}\right)$.

The last term is singular, but integrable at $s=0$, and analytic elsewhere. Thus, $k^{\prime}(t, s)$ has only a singularity of order $s^{-1 / 2}$, and is analytic elsewhere. This shows that $K^{\prime}(\sigma)$ is a compact family of operators, analytic on $\sigma$.

Step 2: Vanishing of the operator as $\Im \sigma \rightarrow-\infty$

To show the kernel vanishes as $\Im \sigma \rightarrow-\infty$, we shift the contour as follows. We break up the integral in (3.19) as follows:

$$
\begin{aligned}
\int_{\mathbb{R}+0 i} \frac{e^{\sigma p}}{1-} e^{\omega p-i \omega s} & \left.\exp \left(\frac{(c(t)-c(t-s))^{2}}{p}\right)-1\right] \frac{d p}{\sqrt{p}} \\
= & \int_{[-\infty-i \epsilon,-i \epsilon]}+\int_{\left[-i \epsilon+0^{-}, 0^{-}\right]}+\int_{\left[0^{+},-i \epsilon+0^{+}\right]}+\int_{[-i \epsilon, \infty-i \epsilon]} \\
& \frac{e^{\sigma p}}{1-e^{\omega p-i \omega s}}\left[\exp \left(\frac{(c(t)-c(t-s))^{2}}{p}\right)-1\right] \frac{d p}{\sqrt{p}}+\text { Residues }
\end{aligned}
$$

The residues take the form $\frac{2 \pi i}{\omega} e^{i \sigma s}\left[\exp \left(\frac{(c(t)-c(t-s))^{2}}{i s}\right)-1\right] \frac{1}{\sqrt{i s}}$ for $0>$ $\Im s>-\epsilon$, and hence decay exponentially. Similarly, the integrals over $[-\infty-$ $i \epsilon,-i \epsilon]$ and $[-i \epsilon, \infty-i \epsilon]$ decay at least as fast as $e^{-\epsilon|\Im \sigma|}$. The integrals over the small region are simply Laplace-type integrals, and can be bounded by:

$$
\begin{aligned}
& \mid \int_{[0,-i \epsilon]} \frac{e^{\sigma p}}{1-e^{\omega p-i \omega s}}\left[\exp \left(\frac{(c(t)-c(t-s))^{2}}{p}\right)-1\right] \frac{d p}{\sqrt{p}} \mid \\
& \leq C \int_{[0, \epsilon]} e^{-\Im \sigma z} \frac{d z}{\sqrt{z}} \leq C|\Im \sigma|^{-1 / 2}
\end{aligned}
$$

Thus for $s \neq 0, k^{\prime}(t, s) \rightarrow 0$ as $\Im \sigma \rightarrow-\infty$, hence $K^{\prime}(\sigma)$ vanishes by Fatou's lemma.

Step 3: Continuation of $K(\sigma)$ 
To show that $K^{\prime}(\sigma)=K(\sigma)$ if $\Im \sigma>0$, we simply move the contour of integration in (3.18b) upward and collect residues:

$$
\begin{aligned}
\int_{\mathbb{R}+0 i} & \frac{e^{\sigma p}}{1-e^{\omega p-i \omega s}}\left[\exp \left(\frac{(c(t)-c(t-s))^{2}}{p}\right)-1\right] \frac{d p}{\sqrt{p}} \\
= & \lim _{N \rightarrow \infty}\left[\int_{\mathbb{R}+i 2 \pi N / \omega} \frac{e^{\sigma p}}{1-e^{\omega p-i \omega s}}\left[\exp \left(\frac{(c(t)-c(t-s))^{2}}{p}\right)-1\right] \frac{d p}{\sqrt{p}}\right. \\
+ & \left.\sum_{j=0}^{N} \frac{2 \pi i}{\omega} e^{i \sigma(s+2 \pi j / \omega)}\left[\exp \left(\frac{(c(t)-c(t-s))^{2}}{i(s+2 \pi j / \omega)}\right)-1\right] \frac{1}{\sqrt{i(s+2 \pi j / \omega)}}\right] \\
& =\sum_{j=0}^{\infty} \frac{2 \pi i}{\omega} e^{i \sigma(s+2 \pi j / \omega)}\left[\exp \left(\frac{(c(t)-c(t-s))^{2}}{i(s+2 \pi j / \omega)}\right)-1\right] \frac{1}{\sqrt{i(s+2 \pi j / \omega}}
\end{aligned}
$$

We then integrate this kernel against an $L^{2}\left(S^{1}, d t\right)$ function $f(t)$ and obtain:

$$
\begin{aligned}
& \int_{0}^{2 \pi / \omega} \sqrt{\frac{i}{\pi}} \frac{\omega}{2 \pi i} \sum_{j=0}^{\infty} \\
& \begin{aligned}
\frac{2 \pi i}{\omega} e^{i \sigma(s+2 \pi j / \omega)} & {\left[\exp \left(\frac{(c(t)-c(t-s))^{2}}{i(s+2 \pi j / \omega)}\right)-1\right] \frac{1}{\sqrt{i(s+2 \pi j / \omega}} f(t-s) d s } \\
& =\sqrt{\frac{i}{\pi}} \int_{0}^{\infty}\left[\exp \left(i \frac{(c(t)-c(t-s))^{2}}{s}\right)-1\right] e^{i \sigma s} f(t-s) \frac{d s}{\sqrt{s}}
\end{aligned}
\end{aligned}
$$

This is in agreement with (3.16). Hence, $K^{\prime}(\sigma)=K_{L}(\sigma)$ for $\Im \sigma>0, \Re \sigma \in(0, \omega)$ and therefore $K^{\prime}(\sigma)$ is the analytic continuation of $K_{L}(\sigma)$.

Now that it is justified, we write $K^{\prime}=K_{L}$. In addition, now that $K_{L}(\sigma)$ and $K_{F}(\sigma)$ are defined, it is clear that $K_{F}(\sigma)+K_{L}(\sigma)=K(\sigma)$.

We now show that $K(\sigma)$ has no more than exponential growth as $\Re \sigma \rightarrow \pm \infty$.

Proposition $3.11 K(\sigma)$ satisfies the following bounds:

$$
\|K(\sigma)\|_{\mathcal{L}\left(l^{2}, l^{2}\right)} \leq C e^{(2 \pi / \omega)|\Im \sigma|}
$$

In addition, as $\Im \sigma \rightarrow+\infty,\|K(\sigma)\|_{\mathcal{L}\left(l^{2}, l^{2}\right)} \rightarrow 0$.

Proof. First, note that $K_{F}(\sigma)$ is trivially bounded as $\Im \sigma \rightarrow \pm-\infty$.

We return to (3.19) to compute a bound on $K_{L}(\sigma)$. Note that the kernel $k^{\prime}(t, s)$ is exponentially bounded. This follows because $k^{\prime}(t, s)$ can be written as:

$$
\begin{array}{r}
\left(\underline{3.19)}=\int_{\mathbb{R} \backslash[-2 \pi / \omega, 2 \pi / \omega]} \frac{e^{\sigma p}}{1-e^{\omega p-i \omega s}}\left[\exp \left(\frac{(c(t)-c(t-s))^{2}}{p}\right)-1\right] \frac{d p}{\sqrt{p}}\right. \\
+\int_{|p|=2 \pi / \omega, \Im p>0} \frac{e^{\sigma p}}{1-e^{\omega p-i \omega s}}\left[\exp \left(\frac{(c(t)-c(t-s))^{2}}{p}\right)-1\right] \frac{d p}{\sqrt{p}} \\
+\frac{2 \pi i}{\omega} e^{i \sigma s}\left[\exp \left(\frac{(c(t)-c(t-s))^{2}}{i s}\right)-1\right] \frac{1}{\sqrt{i s}}
\end{array}
$$


The first integrand is uniformly bounded, independent of $\Im \sigma$. The second integral term is the integral over a compact region, which has a maximum modulus equal to $C e^{(2 \pi / \omega) \Im \sigma}$ at $p=i 2 \pi / \omega$ (with $C$ depending on the rest of the integral). The last term is similarly bounded.

To show that $K(\sigma)$ vanishes as $\Im \sigma \rightarrow \pm \infty$, we simply examine (3.16) and apply the dominated convergence theorem.

We now observe that since $K(\sigma)$ is a compact operator on $L^{2}\left(S^{1}, d t\right)$, the Fredholm alternative applies to $(1-K(\sigma))^{-1}$. Therefore, it makes sense to study properties of solutions to the homogeneous equation $K(\sigma) f(t)=f(t)$.

Proposition 3.12 Suppose $f(t)=K(\sigma) f(t)$ for some $\sigma \in(0, \omega)$. Then $y\left(e^{i \omega t}\right)$ satisfies the equation (the integral must now be interpreted as improper):

$$
y\left(e^{i \omega t}\right)=\sqrt{\frac{i}{\pi}} \int_{0}^{\infty} \exp \left(i \frac{(c(t)-c(t-s))^{2}}{4 s}\right) e^{i \sigma_{b} s} y\left(e^{i \omega(t-s)}\right) \frac{d s}{\sqrt{s}}
$$

Proof. This follows from the definition of $K(\sigma)$.

We have now shown that $K(\sigma): L^{2}\left(S^{1}, d t\right) \rightarrow L^{2}\left(S^{1}, d t\right)$ is an analytic (in $\sigma)$ family of compact operators. This allows us to construct the resolvent.

Proposition 3.13 Suppose $(1-K(\sigma))^{-1}$ has a pole of multiplicity $n$ at a point $\sigma_{b}$. Then near $\sigma_{b}$ :

$$
(1-K(\sigma))^{-1}=\sum_{j=1}^{n} \frac{y(t)\langle y(t) \mid \cdot\rangle}{\left(\sigma-\sigma_{b}\right)^{j}}+D(\sigma)
$$

where $D(\sigma)$ is analytic near $\sigma_{b} . y_{\sigma_{b}, j}(t)$ solves $\left(1-K\left(\sigma_{b}\right)\right) y_{\sigma_{b}, j}(t)=0$. The functions $y(t)$ is an $L^{2}\left(S^{1}\right)$ function.

If $\sigma_{b}=0$, then the same result holds, except that the poles are in the variable $\sqrt{\sigma}$ instead of $\left(\sigma-\sigma_{b}\right)$.

Proof. This is merely the analytic Fredholm alternative theorem. There is only one technical point regarding the behavior near $\sigma=0$ due to the fact that $K(\sigma)$ is singular there.

This can be remedied as follows. The function $y(\sigma, t)$ satisfies the following equation:

$$
\left(1-K_{F}(\sigma)-K_{L}(\sigma)\right) y(\sigma, t)=y_{0}(\sigma, t)
$$

We expand $K_{F}(\sigma) y(\sigma, t)$ as in the proof of Proposition 3.8 Due to the fact that $K_{F}(\sigma)$ is singular only in the 0 'th Fourier component (see (3.15)), we find that:

$$
\begin{aligned}
\left(1-K_{F}(\sigma)\left(1-P_{0}\right)-K_{L}(\sigma)\right) y(\sigma, t) & +\sigma^{-1 / 2} P_{0} y(\sigma, t) \\
& =\sigma^{-1 / 2}(1 / 2) \int_{\mathbb{R}} \psi_{0}(x) d x+f\left(\sigma^{1 / 2}, t\right)
\end{aligned}
$$


Here, $P_{0}$ is the projection onto the 0 'th Fourier coefficient of a function. Take as an ansatz that $P_{0} y(0, t)=(1 / 2) \int_{\mathbb{R}} \psi_{0}(x) d x$. Then, since $K_{F}(\sigma)(1-$ $\left.P_{0}\right)-K_{L}(\sigma)$ is compact and analytic in $\sigma^{1 / 2}$, we find that

$$
y(\sigma, t)=\left[1-K_{F}(\sigma)\left(1-P_{0}\right)-K_{L}(\sigma)\right]^{-1} f\left(\sigma^{1 / 2}, t\right)
$$

is meromorphic in $\sigma^{1 / 2}$. This implies that our ansatz was consistent.

Proposition $3.14(1-K(\sigma))^{-1}$ has precisely one pole, counting multiplicities. Therefore, it can be decomposed as

$$
(1-K(\sigma))^{-1}=\frac{Y_{b}(t)\left\langle Y_{b}(t) \mid \cdot\right\rangle}{\sigma-\sigma_{b}}+D(\sigma, t)
$$

when $\sigma_{b} \neq 0$, or

$$
(1-K(\sigma))^{-1}=\frac{Y_{b}(t)\left\langle Y_{b}(t) \mid \cdot\right\rangle}{\sqrt{\sigma}}+D(\sigma, t)
$$

when $\sigma_{b}=0 . D(\sigma, t)$ is analytic in $\sigma^{1 / 2}$.

Proof. This result is the analytic implicit function theorem, applied to compact analytic operators. One can find a precise proof in [20, page 368-370] where that result is theorems 1.7 and 1.8 (see also the discussion following theorem 1.7).

\subsection{Time behavior of $\psi(x, t)$}

We have now shown that $K(\sigma)$ is a compact analytic operator. By the Fredholm alternative, $(1-K(\sigma))^{-1}$ is a meromorphic operator family. By deforming the contour in (3.6a), we can determine the behavior of $Y(t)$. Once this is complete, we can calculate $\psi_{b}(x, t)$ and $\psi_{d}(x, t)$ and finish the proof of Theorem 1

Lemma 3.15 Let $(1-K(\sigma))^{-1}$ have a pole at the point $\sigma_{b}=\alpha+i \gamma \neq 0$. Then $Y(t)$ can be written as:

$$
Y(t)=e^{-i \sigma_{b} t} Y_{b}(t)+D(t)
$$

with $Y_{b}(t)$ the residue at $\sigma_{b}$. The function $D(t)$ is given by:

$$
D(t)=\sum_{n \in \mathbb{Z}} e^{-i n \omega t} \mathcal{L} \mathcal{B} \sum_{j=3}^{\infty} D_{j, n} t^{-j / 2}
$$

where $\mathcal{L B}$ is the Borel summation operator. The sum over $j$ is convergent in $l^{1}$. This shows that $|D(t)| \leq C /\left\langle t^{-3 / 2}\right\rangle$ (where $\langle x\rangle=\sqrt{1+x^{2}}$ ).

Supposing that $\sigma_{b}=0, Y(t)=D(t)$ except that in (3.29) the sum starts at $j=1$ rather than $j=3$. 
Proof. Because $(1-K(\sigma))^{-1}$ is meromorphic in $\sigma, y(\sigma, t)$ can be written as

$$
y(\sigma, t)=(1-K(\sigma))^{-1} y_{0}(\sigma, t)=\frac{Y_{b}(t)\left\langle Y_{b}(t) \mid y_{0}\left(\sigma_{b}, t\right)\right\rangle}{\sigma-\sigma_{b}}+D(\sigma) y_{0}(\sigma, t)
$$

We compute $Y(t)$ using (3.6a), and shifting the contour:

$$
\begin{aligned}
Y(t)= & \omega^{-1} \int_{0_{+}}^{\omega_{-}} e^{-i \sigma t} y(\sigma, t) d \sigma \\
& =\omega^{-1} \int_{0_{+}}^{-i M+0_{+}} e^{-i \sigma t} y(\sigma, t) d \sigma+\omega^{-1} \int_{-i M}^{-i M+\omega} e^{-i \sigma t} y(\sigma, t) d \sigma \\
+\omega^{-1} \int_{-i M+\omega_{-}}^{\omega_{-}} e^{-i \sigma t} y(\sigma, t) d \sigma+\text { Residues }=\omega^{-1} \int_{-i M}^{-i M+\omega} e^{-i \sigma t} y(\sigma, t) d \sigma & \\
& +\omega^{-1} \int_{0}^{-i M+0} e^{-i \sigma t} y\left(\sigma+0_{+}, t\right)-e^{-\left(i \sigma+\omega_{-}\right) t} y\left(\sigma+\omega_{-}, t\right) d \sigma \\
& \quad+\text { Residues }
\end{aligned}
$$

We integrate from $0+$ to $\omega$ - due to the singularity at $\sigma=0$. The residue term is given by $(2 \pi / \omega) i e^{-i \sigma_{b} t} Y_{b}(t)\left\langle Y_{b}(t) \mid y_{0}(\sigma, t)\right\rangle 1$, if $M>-\Im \sigma_{b}$.

To show Borel Summability, we must show that $D(t)$ is the Laplace transform of an analytic function (in particular analytic in $\sigma^{1 / 2}$ ). We do this as follows.

We take the limit as $M \rightarrow \infty$ (justified shortly). By (3.6c), we can change the integral in the last line of (3.31) to:

$$
\omega^{-1} \int_{0}^{-i \infty} e^{-i \sigma t}\left(y\left(\sigma+0_{+}, t\right)-y\left(\sigma+0_{-}, t\right)\right) d \sigma
$$

Note that $y(\sigma, t)$ is analytic in $\sigma^{1 / 2}$, and thus $y\left(\sigma+0_{+}, t\right)-y\left(\sigma+0_{-}, t\right)$ can be expanded in a Puiseux series in $\sigma^{1 / 2}$ (and a Fourier series in $t$ ). Watson's lemma yields:

$$
\begin{aligned}
\text { (3.32) }=\omega^{-1} \int_{0}^{-i \infty} e^{-i \sigma t} \sum_{n \in \mathbb{Z}} e^{-i n \omega t} & \sum_{j=0}^{\infty} D_{j, n} \sigma^{n / 2} d \sigma \\
& \sim \omega^{-1} \sum_{n \in \mathbb{Z}} e^{i n \omega t} \sum_{j=3}^{\infty} D_{j, n} \Gamma(j / 2) t^{-j / 2}
\end{aligned}
$$

This is what we wanted to show.

When $\sigma_{b}=0$, the result follows simply by noting that the sum over $j$ in (3.32) starts from $j=-1$ rather than $j=0$, thereby letting the sum on the right of (3.32) start at $j=1$ instead of $j=3$.

It remains to show that we can take the limit as $M \rightarrow \infty$. Begin by writing $y(\sigma, t)=(1-K(\sigma))^{-1} y_{0}(\sigma, t)$. By Proposition $3.10 K(\sigma) \rightarrow 0$ as $\Im \sigma \rightarrow-\infty$. Thus, by the Neumann series, $(1-K(\sigma))^{-1} \rightarrow 1$ (and hence is uniformly 
bounded, say by $C$ ), and then

$$
\left|\int_{-i M}^{-i M+\omega} e^{-i \sigma t} y(\sigma, t) d \sigma\right| \leq C \int_{-i M}^{-i M+\omega} e^{-|\Im \sigma| t}\left|y_{0}(\sigma, t)\right| d \sigma \leq C e^{-|\Im \sigma| t} e^{C_{2}|\Im \sigma|}
$$

with $C_{2}$ given in Proposition 3.5 Thus for $t>C_{2}$ we find that this term vanishes as $M \rightarrow \infty$.

We now reconstruct $\psi(x, t)$ in the velocity gauge. The basic idea is as follows. We know that $\psi_{v}(c(t), t)=D(t)+e^{-i \sigma_{b} t} Y_{b}(t)$. Using the fact that $\delta(x-c(t)) \psi_{v}(x, t)=\delta(x-c(t)) \psi_{v}(c(t), t)$, we find that $\psi_{v}(x, t)$ satisfies the following equation:

$$
\begin{aligned}
& i \partial_{t} \psi_{v}(x, t)=-\partial_{x}^{2} \psi_{v}(x, t)-2 \delta(x-c(t)) \psi_{v}(x, t) \\
&=\partial_{x}^{2} \psi_{v}(x, t)-2 \delta(x-c(t)) \psi_{v}(c(t), t) \\
&=-\partial_{x}^{2} \psi_{v}(x, t)-2 \delta(x-c(t))\left[D(t)+e^{-i \sigma_{b} t} Y_{b}(t)\right]
\end{aligned}
$$

Assuming that $D(t)$ and $Y_{b}(t)$ are known, this can be solved by Duhamel's principle. We break it into pieces, and do exactly that.

We first construct the dispersive part.

Proposition 3.16 Let $\psi_{d}(x, t)$ satisfy:

$$
i \partial_{t} \psi_{d}(x, t)=-\partial_{x}^{2} \psi_{d}(x, t)-2 \delta(x-c(t)) D(t)
$$

with initial condition:

$$
\int_{0}^{\omega} \sqrt{\frac{i}{\pi}} \int_{0}^{\infty} \exp \left(\frac{i(x-c(0-s))^{2}}{4 s}\right) e^{i \sigma s} D(\sigma,-s) \frac{d s}{\sqrt{s}} d \sigma
$$

Then $\psi_{d}(x, t)$ is Borel summable in the following sense. There exists a family of functions $D_{j, n}(x)$ so that:

$$
\psi_{d}(x, t)=\sum_{n \in \mathbb{Z}} e^{-i n \omega t} \mathcal{L B} \sum_{j=3}^{\infty} D_{j, n}(x) \Gamma(j / 2) t^{-j / 2}
$$

In addition, $\psi_{d}(x, t)$ is uniformly bounded in $L^{2}$, that is:

$$
\sup _{t}\left\|\psi_{d}(x, t)\right\|_{L^{2}(\mathbb{R}, d x)}<\infty
$$

\section{Proof.}

Borel Summability:

To show Borel summability, we must show that for each $x, \psi_{d}(x, t)$ is the Laplace transform of an analytic function. 
Begin by observing that $\psi_{v}(x, t)$ satisfies (3.2). By the same argument as in Proposition 3.7 we find that:

$$
\begin{aligned}
& \mathcal{Z}\left[\psi_{v}(x, \cdot)\right](\sigma, t)=\mathcal{Z}\left[\psi_{v, 0}(x, \cdot)\right](\sigma, t) \\
&+\sqrt{\frac{i}{\pi}} \int_{0}^{\infty} \exp \left(\frac{i(x-c(t-s))^{2}}{4 s}\right) e^{i \sigma s} \mathcal{Z}[D(\cdot)](\sigma, t-s) \frac{d s}{\sqrt{s}}=\mathcal{Z}\left[\psi_{v, 0}(x, \cdot)\right](\sigma, t) \\
&+\sqrt{\frac{i}{\pi}} \int_{0}^{\infty} \exp \left(\frac{i(x-c(t-s))^{2}}{4 s}\right) e^{i \sigma s} D(\sigma, t-s) \frac{d s}{\sqrt{s}}
\end{aligned}
$$

Let $K(x, \sigma)$ denote the integral operator in (3.38). By the same arguments as in Section 3.2 we find that $K(x, \sigma)$ is analytic in $\sigma^{1 / 2}$. In particular, we find that $K(x, \sigma)$ can be decomposed as:

$$
\begin{aligned}
K(x, \sigma) D(\sigma, t) & =\sum_{n \in \mathbb{Z}} \frac{D_{n}(\sigma) e^{-i n \omega t}}{\sqrt{\sigma+n \omega}} \\
& +\sqrt{\frac{i}{\pi}} \int_{0}^{\infty} \exp \left[\left(\frac{i(x-c(t-s))^{2}}{4 s}\right)-1\right] e^{i \sigma s} D(\sigma, t-s) \frac{d s}{\sqrt{s}}
\end{aligned}
$$

Here, $D_{n}(\sigma)$ is the $n$ 'th Fourier coefficient of $D(\sigma, t)$. Since $D(\sigma, t)$ is analytic in $\sigma^{1 / 2}$, the $D_{n}(\sigma)$ 's are as well.

By the argument given in the proof of Proposition 3.13 we find that $D_{0}(\sigma)=$ $(-1 / 2) \int_{\mathbb{R}} \psi_{0}(x) d x$. Thus, we find that $\mathcal{Z}\left[\psi_{v}(x, \cdot)\right](\sigma, t)$ is actually analytic in $\sigma^{1 / 2}$, since the $\sigma^{-1 / 2}$ terms of $\mathcal{Z}\left[\psi_{v, 0}(x, \cdot)\right](\sigma, t)$ and $K(x, \sigma) D(\sigma, t)$ cancel.

Proceeding the same way as in Proposition 3.15 we find that $\psi(x, t)$ is also the Borel sum of a series in $t^{-n / 2}$. The initial condition is satisfied because it is merely the inverse Zak transform of $K(x, \sigma) D(\sigma, t)$ evaluated at $t=0$. The function is Laplace transformable because $D(\sigma, t)$ is, and because (by the same argument as in Proposition 3.10) the integral operator $K(x, \sigma)$ decays as $\Im \sigma \rightarrow-\infty$.

Uniform Boundedness in $L^{2}$ :

We write using Duhamel's principle:

$$
\psi_{d}(x, t)=e^{i \partial_{x}^{2} t} \psi_{d}(x, 0)-2 i \int_{0}^{t} e^{i \partial_{x}^{2}(t-s)} \delta(x-c(s)) D(s) d s
$$

Considering only the integral term (the first term is clearly $L^{2}$ ), we Fourier transform in space and integrate by parts in time to obtain:

$$
\begin{gathered}
\int_{0}^{t} e^{-i k^{2}(t-s)} e^{i c(s) k} D(s) d s=e^{-i k^{2} t} \int_{0}^{t} e^{i k^{2} s} e^{i c(s) k} D(s) d s \\
=e^{-i k^{2} t}\left[\frac{e^{i k^{2} s}}{i k^{2}} e^{i c(s) k} D(s)\right]_{s=0}^{t} \\
-e^{-i k^{2} t} \int_{0}^{t} \frac{e^{i k^{2} s}}{i k^{2}}\left(i b(s) k e^{i c(s) k} D(s)+e^{i c(s) k} D^{\prime}(s)\right) d s
\end{gathered}
$$


Since $D(s)$ and $D^{\prime}(s)$ decay like $s^{-3 / 2}$ for large $s$ and are smooth, the integral in (3.39) is manifestly square integrable for $|k|>1^{4}$ since it decays at least as fast as $k^{-1}$.

For $|k| \leq 1$, we observe that

$$
\left|e^{i k^{2} t} \int_{0}^{t} e^{i k^{2} s} e^{i c(s) k} D(s) d s\right| \leq C \int_{0}^{t} D(s) d s \leq C
$$

is uniformly bounded and therefore square integrable on compact sets. Thus, we have shown that $\psi_{d}(x, t)$ is square integrable, uniformly in time.

We now construct the resonance or bound state. In addition, we show that $\Im \sigma_{b} \leq 0$.

Proposition 3.17 Fix $\sigma_{b}$. Let $Y_{b}(t)$ be the periodic part of $Y(t)$, as constructed in Proposition 3.15. Then there exists a function $\psi_{b}(x, t)$ in the space $L^{2}\left([0,2 \pi / \omega] ; H^{1}(\mathbb{R})\right)$ such that $\psi_{b}(0, t)=Y_{b}(t)$ and such that $\varphi_{b}(x, t)$ satisfies:

$$
i \partial_{t}\left(e^{-i \sigma_{b} t} \psi_{b}(x, t)\right)=-\partial_{x}^{2} e^{-i \sigma_{b} t} \psi_{b}(x, t)-2 \delta(x-c(t)) e^{-i \sigma_{b} t} Y_{b}(t)
$$

In particular, we show that it is impossible that $\Im \sigma_{b}>0$.

Proof. We can solve (3.40) by Duhamel:

$$
e^{-i \sigma_{b} t} \psi_{b}(x, t)=e^{-i \partial_{x}^{2} t} \psi_{b}(x, 0)-2 i \int_{0}^{t} e^{i \partial_{x}^{2}(t-s)} \delta(x-c(s)) e^{-i \sigma_{b} s} Y_{b}(s) d s
$$

Let $f(x, t)$ denote the integral term in (3.41). After Fourier transforming in time, $\hat{f}(k, t)$ is given by:

$$
\hat{f}(k, t)=-2 \int_{0}^{t} e^{-i k^{2}(t-s)} e^{-i \sigma s} e^{i k c(s)} Y_{b}(s) d s
$$

We substitute the following decomposition

$$
e^{i k c(s)} Y_{b}(s)=\sum_{n \in \mathbb{Z}} h_{n}(k) e^{-i n \omega s}
$$

into (3.42) and compute the integral. We separate the problem into three cases, depending on the imaginary part of $\sigma_{b}$.

Observe that for any $n, h_{n}(k)=\left\langle e^{-i n \omega t} \mid e^{-i k c(t)} Y_{b}(t)\right\rangle$ is an entire function in $k$.

Case 1: $\Im \sigma_{b}<0$

We do a similar calculation as above, yielding:

$$
\begin{aligned}
& \hat{f}(k, t)=e^{-i k^{2} t} \int_{0}^{t} e^{i\left(k^{2}-\sigma_{b}\right) s} \sum_{n \in \mathbb{Z}} h_{n}(k) e^{-i n \omega s} d s \\
& =e^{-i \sigma_{b} t} \sum_{n \in \mathbb{Z}} h_{n}(k) \frac{e^{-i n \omega t}}{i\left(k^{2}-\sigma_{b}-n \omega\right)}-e^{-i k^{2} t} \sum_{n \in \mathbb{Z}} h_{n}(k) \frac{1}{i\left(k^{2}-\sigma_{b}-n \omega\right)}
\end{aligned}
$$

\footnotetext{
${ }^{4}$ the number 1 is arbitrary, and could be any number bigger than 0
} 
This is well defined, since the denominator is never zero.

Case 2: $\Im \sigma_{b}>0$ :

This is impossible. Eq. (3.44) is still valid, except now $e^{-i \sigma_{b} t} \psi_{b}(x, t)$ and hence $\psi(x, t)$ is growing exponentially with time. This contradicts unitary evolution.

Case 3: $\Im \sigma_{b}=0$ :

We compute $\hat{f}(k, t)$ as in (3.44). However, the denominator $i\left(k^{2}-\sigma_{b}-n \omega\right)$ is now zero when $k= \pm \sqrt{\sigma_{b}+n \omega}$ (for $\sigma_{b}+n \omega>0$ ). We will show that if the numerator is not zero at these points, then unitary evolution does not hold.

We rewrite (3.44) as:

$$
\begin{aligned}
\hat{f}(k, t)=e^{-i k^{2} t} \sum_{n \in \mathbb{Z}} h_{n}(k) \frac{e^{i\left(k^{2}-\sigma_{b}-n \omega\right) t}-1}{i\left(k^{2}-\sigma_{b}-n \omega\right)} & \\
=i e^{-i k^{2} t} \sum_{n \in \mathbb{Z}} h_{n}(k) \frac{1-\cos \left(\left(k^{2}-\sigma_{b}-n \omega\right) t\right)}{\left(k^{2}-\sigma_{b}-n \omega\right)} & +e^{-i k^{2} t} \sum_{n \in \mathbb{Z}} h_{n}(k) \frac{\sin \left(\left(k^{2}-\sigma_{b}-n \omega\right) t\right)}{\left(k^{2}-\sigma_{b}-n \omega\right)}
\end{aligned}
$$

Note that for some constants $C, C^{\prime}$ :

$$
\lim _{t \rightarrow \infty} \frac{\sin \left(\left(k^{2}-\sigma_{b}-n \omega\right) t\right)}{\left(k^{2}-\sigma_{b}-n \omega\right)}=C \delta\left(k-\sqrt{\sigma_{b}+n \omega}\right)+C^{\prime} \delta\left(k+\sqrt{\sigma_{b}+n \omega}\right)
$$

as $t \rightarrow \infty$. Thus, $\hat{f}(k, t)$ is approaching a $\delta$-function at $k= \pm \sqrt{\sigma_{b}+n \omega}$. Therefore, unless $h_{n}\left( \pm \sqrt{\sigma_{b}+n \omega}\right)=0$, unitary evolution does not hold since $\|\hat{f}(k, t)\|_{L^{2}}$ grows without bound.

In physical terms, plane waves are being radiated which increases the probability of the wavefunction.

This can be made precise by integrating against a smooth bump function $b(k)$ which has a $k^{-1 / 2+\delta}$ singularity at $k= \pm \sqrt{\sigma_{b}+n \omega}$. Since the bump function is in $L^{2}, \sup _{t}|\langle b(k) \mid \hat{f}(k, t)\rangle|=b\left(\sqrt{\sigma_{b}+n \omega}\right)=\infty \leq C\|\hat{f}(k, t)\|_{L^{2}}$, a contradiction. Thus we have arrived at a contradiction unless $h_{n}\left( \pm \sqrt{\sigma_{b}+n \omega}\right)=0$.

Conclusion

We thus define $\hat{\psi}_{b}(k, t)$ by:

$$
\hat{\psi}_{b}(k, t)=\sum_{n \in \mathbb{Z}} h_{n}(k) \frac{e^{-i n \omega t}}{i\left(k^{2}-\sigma_{b}-n \omega\right)}
$$

Note that $e^{-i k^{2} t} e^{-i \sigma_{b} t} \hat{\psi}_{b}(k, 0)+\hat{f}(k, t)=e^{-i \sigma_{b} t} \hat{\psi}_{b}(k, t)$, and thus $e^{-i \sigma_{b} t} \psi_{b}(x, t)$ solves (3.40).

Lemma 3.18 Suppose $\Im \sigma_{b}=0$. Then $\psi_{b}(0, t)=Y_{b}(t)$ and $\psi_{b}(x, t)$ satisfies:

$$
\left(-i \partial_{t}-\partial_{x}^{2}-2 \delta(x-c(t))\right) \psi_{b}(x, t)=\sigma_{b} \psi_{b}(x, t)
$$


Proof. Note that $\psi_{b}(x, t)$ is given by:

$$
e^{-i \sigma_{b} t} \psi_{b}(x, t)=-2 i \int_{0}^{\infty} e^{i \partial_{x} s} \delta(x-c(t-s)) e^{-i \sigma_{b}(t-s)} Y_{b}(t-s) d s
$$

Evaluating this at $x=c(t)$ yields:

$$
e^{-i \sigma_{b} t} \psi_{b}(c(t), t)=e^{-i \sigma_{b} t} K\left(\sigma_{b}\right) Y_{b}(t)
$$

Thus, $\delta(x-c(t)) \psi_{b}(c(t), t)=\delta(x-c(t)) Y_{b}(t)$, and since $\psi_{b}(x, t)$ satisfies (3.40), it must also satisfy (3.46).

We have now proved Theorem 1

\section{Concluding Remarks}

In this paper we studied the interaction of a simple model atom with a dipole radiation field of arbitrary strength. We obtained a resonance expansion, in which resonances can be resolved regardless of their complex quasi-energy. In particular, we obtained a rigorous definition of the ionization rate $\gamma=-2 \Im \sigma_{b}$ and Stark-shifted energy, $\Re \sigma_{b}$.

We applied this result to show that complete ionization occurs $(\gamma>0)$ when $E(t)$ is a trigonometric polynomial.

We conclude by discussing possible future directions of research.

\subsection{Perturbative and numerical calculations}

The main feature of our method is that it turns a time dependent problem on $\mathbb{R}$ into a compact analytic Fredholm integral equation. This implies that a family of finite dimensional approximations can be used (in the Zak domain) to approximate solutions to the time dependent Schrödinger equation. We have carried out perturbative calculations in this manner, recovering Fermi's Golden Rule and the multiphoton effect.

We believe that the quasi-energy methodology used here and in related papers [6, 10, 9] can be used for quantitative calculations of interesting physical phenomenon. Phenomena which we believe can be treated by our methods include LICS (Laser Induced Continuum States) 30, 19, High Harmonic Generation 23, 22, multiphoton ionization [5, 25] and others. It is our aim to develop an efficient calculation method based on our formalism and obtain quantitative results for some of the physical phenomena mentioned above.

\subsection{Resonance theory}

Significant effort has been devoted to the rigorous definition of resonances and quasimodes, especially in cases when the scattering matrix is unavailable. The best results we are aware of are those of [18, 31, based on complex scaling, and those based on analytic continuation of the S-matrix, e.g. 2]. We provide an 
alternative definition: a quasi-bound state is the coefficient of an exponentially decaying term in the asymptotic expansion for $\psi(x, t)$ near $t=\infty$. We aim to study the consequences of this definition, and determine whether it is compatible with other definitions, and hopefully use it to provide a more complete picture of the time evolution of $\psi(x, t)$.

\subsection{Extension to 3 dimensions}

In the case of $H_{0}=-\Delta-2 \delta(\overrightarrow{\vec{x}})$ with $\vec{x} \in \mathbb{R}^{3}$, a similar equation to (3.4) can be derived. Due to the fact that $\delta(\vec{x})$ is not in $H^{-1}\left(\mathbb{R}^{3}\right), \psi(\vec{x}, t)$ develops singularities in finite time. However, $\psi(\vec{x}, t)$ can be decomposed as $\psi(\vec{x}, t)=Y(t) /|\vec{x}|+\psi_{c}(\vec{x}, t)$ where $\psi_{c}(\vec{x}, t)$ is continuous and $\psi_{c}(0, t)=Y(t)^{5}$, with $Y(t)$ satisfying an integral equation similar to (3.4) (see [14]). The kernel even satisfies the property of being of exponential order two, so that Proposition 2.2 is likely to hold.

For this reason, we believe most of our results can be adapted to the threedimensional case (although we do not intent to actually pursue this). This belief is strengthened by the fact that similar results have been extended in the past. The paper [10 proves an ionization result similar to this one, using similar methods, but treating the system with time dependent Hamiltonian $H(t)=-\Delta-(2+c(t)) \delta(x)$, which [6] extends to 3 dimensions.

\section{A Proof of Proposition 2.1}

Recall the construction of $\hat{\psi}_{b}(k, t)$ given in (3.45) on page 26 We will reconstruct $\psi_{b}(x, t)$ by inverse Fourier transforming (3.45).

$$
\int \hat{\psi}_{b}(k, t) e^{i k x} d k=\sum_{n \in \mathbb{Z}} \int e^{i k x} h_{n}(k) \frac{e^{-i n \omega t}}{i\left(k^{2}-\sigma_{b}-n \omega\right)} d k
$$

Note that $e^{i k x} h_{n}(k)=\left\langle e^{-i n \omega t} \mid e^{i k(x-c(t))} Y_{b}(t)\right\rangle$ decays like $e^{-\Im k(x-c(t))}$ for $x>c(t)$ and $e^{\Im k(c(t)-x)}$ for $x<c(t)$. Therefore we can push the contour of integration upward (when $x>\|c(t)\|_{L^{\infty}}$ ) and downward (when $x<-\|c(t)\|_{L^{\infty}}$ ), and collect residues.

When $\sigma_{b}+n \omega>0$, there are no residues (see the discussion in the proof of Proposition [3.17 case 3) since $h_{n}(k)$ is zero where $k^{2}=\sigma_{b}+n \omega$. Let $M$ denote the least integer for which $\sigma_{b}+n \omega>0$.

Pushing the contour up yields:

$$
\psi_{b}(x, t)= \begin{cases}\sum_{n<M} e^{-i n \omega t} h_{n}\left(-i \sqrt{\left|\sigma_{b}+n \omega\right|}\right) e^{\sqrt{\left|\sigma_{b}+n \omega\right|} x}, & x \leq-\|c(t)\|_{L^{\infty}} \\ \sum_{n<M} e^{-i n \omega t} h_{n}\left(i \sqrt{\left|\sigma_{b}+n \omega\right|}\right) e^{-\sqrt{\left|\sigma_{b}+n \omega\right|} x}, & x \geq\|c(t)\|_{L^{\infty}}\end{cases}
$$

Equating $h_{n}\left(-i \sqrt{\left|\sigma_{b}+n \omega\right|}\right)$ with $\psi_{n,-}$ and $h_{n}\left(-i \sqrt{\left|\sigma_{b}+n \omega\right|}\right)$ with $\psi_{n,+}$ yields the result we seek when $|x|>\|c(t)\|_{L^{\infty}}$.

${ }^{5}$ This implies that all solutions in 3 dimensions are weak solutions, which causes many of the technical difficulties. 
By transforming from the velocity gauge to the magnetic gauge, we can directly continue the results to $x=0$ (corresponding to $x=c(t)$ in the velocity gauge).

\section{B Proof of Proposition 2.2}

We observe that by the results of Section 3 if a bound state exists, then:

$$
\psi_{B}(0, t)=e^{a(t) / 4} e^{-i a(t)} Y_{b}(t)
$$

Setting $z=e^{-i \omega t}$, and $y(z)=Y_{b}(t)$, we wish to show that $y(z)=f(z)+g(z)$ with $f, g$ both entire of exponential order $2 n$. This is equivalent to showing that:

$$
\left|Y_{b}(t+i \alpha)\right| \leq C \exp \left[C^{\prime} \exp (|2 N \omega \alpha|)\right]
$$

The function $Y_{b}(t)$ satisfies the equation:

$$
Y_{b}(t)=\int_{0}^{2 \pi / \omega} k^{\prime}(t, s) Y_{b}(t-s) d s=-\int_{0}^{2 \pi / \omega} k^{\prime}(t, t-s) Y_{b}(s) d s
$$

with $k^{\prime}(t, s)$ as defined in (3.18b). Thus we obtain the bound:

$$
\left|Y_{b}(t+i \alpha)\right| \leq \int_{0}^{2 \pi / \omega}\left|k^{\prime}(t+i \alpha, t+i \alpha-s)\right|\left|Y_{b}(s)\right| d s
$$

and it suffices to bound $\left|k^{\prime}(t+i \alpha, t+i \alpha-s)\right|$. From the definition of $k^{\prime}(t, s)$, we find:

$$
\begin{aligned}
& k^{\prime}(t+i \alpha, t+i \alpha-s) \\
& \quad=\frac{\omega}{2 \pi i} \int_{\mathbb{R}+0 i} \frac{e^{\sigma p}}{1-e^{\omega p+\alpha-i \omega(t-s)}}\left[\exp \left(\frac{(c(t+i \alpha)-c(s))^{2}}{p}\right)-1\right] \frac{d p}{\sqrt{p}}
\end{aligned}
$$

Supposing $\alpha / \omega>1$ (permissible, since we are interested in the behavior as $\alpha \rightarrow \infty)$, then the integrand is analytic for $z=r e^{i \theta}, 0<r<1$ and $0 \leq \theta \leq \pi$. Thus, we can deform the contour from $\mathbb{R}+0 i$ to $\gamma=\partial\{z: \Im z<0$ or $|z|<1\}$.

Note that for some constant $C,|c(t+i \alpha)| \leq C e^{N \omega|\alpha|}$, since $c(t)$ is a trigonometric polynomial of order $N$.

We find that there are 3 regions of integration which contribute to $k^{\prime}(t+$ $i \alpha, t+i \alpha-s)$. The regions of integration contributing come from the region near $1-e^{\omega p+\alpha-i \omega(t-s)}=0$ (the pole of the integrand), large $p$ and small $p$.

If the pole is closer to $\mathbb{R}$ than $\pi / \omega$, we deform $\gamma$ up to encircle it, staying at a distance piw away from it. Otherwise, we ignore it. Therefore, in any case, for $z \in \gamma, 1-e^{\omega p+\alpha-i \omega(t-s)}$ is uniformly bounded away from zero.

We then split $\gamma=\gamma_{<} \cup \gamma_{>} \cup \gamma_{\alpha}$ where $\gamma_{<}=\left\{p \in \gamma:|p|<\left(C e^{N \omega|\alpha|}+\right.\right.$ $\left.\left.\|c(s)\|_{L^{\infty}}\right)^{2}\right\}$ and $\gamma_{>}=\gamma \backslash \gamma_{<}$. We therefore find that: 


$$
\begin{aligned}
& \left|k^{\prime}(t+i \alpha, t+i \alpha-s)\right| \leq \mid \text { residue } \mid \\
& \quad C \int_{\gamma_{<}}\left|\frac{e^{\sigma p}}{1-e^{\omega p+\alpha-i \omega(t-s)}}\left[\exp \left(\frac{(c(t+i \alpha)-c(s))^{2}}{p}\right)-1\right]\right| \frac{d p}{\sqrt{|p|}} \\
& +C \int_{\gamma_{>}}\left|\frac{e^{\sigma p}}{1-e^{\omega p+\alpha-i \omega(t-s)}}\left[\exp \left(\frac{(c(t+i \alpha)-c(s))^{2}}{p}\right)-1\right]\right| \frac{d p}{\sqrt{|p|}} \leq C
\end{aligned}
$$

The residue can be bounded by:

$$
\begin{aligned}
& \leq C\left|e^{\sigma(-\alpha+i \omega(t-s)) / \omega}\left[\exp \left(\frac{(c(t+i \alpha)-c(s))^{2}}{(-\alpha+i \omega(t-s)) / \omega}\right)-1\right] \frac{1}{\sqrt{(-\alpha+i \omega(t-s)) / \omega}}\right| \\
& \leq C \exp \left(C|c(t+i \alpha)|^{2}\right) \leq C \exp (C \exp (2 N \omega|\alpha|))
\end{aligned}
$$

We bound the integral over the compact region $\gamma_{<}$simply by taking absolute values:

$$
\begin{gathered}
\int_{\gamma_{<}}\left|\frac{e^{\sigma p}}{1-e^{\omega p+\alpha-i \omega(t-s)}}\left[\exp \left(\frac{(c(t+i \alpha)-c(s))^{2}}{p}\right)-1\right]\right| \frac{d p}{\sqrt{|p|}} \\
\leq\left|\gamma_{<}\right| C \exp (C \exp (2 N \omega|\alpha|))
\end{gathered}
$$

For the integral over $\gamma_{>}$, we use the fact that if $|z|<1,\left|e^{z}-1\right| \leq e|z|$ :

$$
\begin{gathered}
\int_{\gamma_{>}}\left|\frac{e^{\sigma p}}{1-e^{\omega p+\alpha-i \omega(t-s)}}\left[\exp \left(\frac{(c(t+i \alpha)-c(s))^{2}}{p}\right)-1\right]\right| \frac{d p}{\sqrt{|p|}} \\
\quad \int_{\gamma_{>}}\left|\frac{e^{\sigma p}}{1-e^{\omega p+\alpha-i \omega(t-s)}} \frac{\left(C e^{N \omega|\alpha|}+\|c(s)\|_{L^{\infty}}\right)^{2}}{|p|}\right| \frac{d p}{\sqrt{|p|}} \\
\quad \leq C e^{2 N \omega|\alpha|} \int_{\gamma_{>}}\left|\frac{e^{\sigma p}}{1-e^{\omega p+\alpha-i \omega(t-s)}} p^{-3 / 2}\right| d p \leq C \exp (C \exp (2 N \omega|\alpha|))
\end{gathered}
$$

Combining these estimates, we find that $k^{\prime}(t+i \alpha, t+i \alpha-s)$ has the required growth as $\alpha \rightarrow \infty$, hence $Y_{b}(t)$ does. The same argument applies as $\alpha \rightarrow-\infty$.

Acknowledgements: We thank A. Soffer and M. Kiessling for useful discussions. J.L.L. and O.C. would like to thank the IHES in Bures-sur-Yvette and the IAS in Princeton where part of the work was done. Work supported by NSF Grants DMS-0100495, DMS-0406193, DMS-0600369, DMS01-00490, DMR 01-279-26 and AFOSR grant AF-FA9550-04. Any opinions, findings, conclusions or recommendations expressed in this material are those of the authors and do not necessarily reflect the views of the National Science Foundation. 


\section{References}

[1] A. Jensen A. Galtbayar and K. Yajima. Local time-decay of solutions to schrodinger equations with time-periodic potentials. J. Stat. Phys., 116:231, 2004.

[2] Shmuel Agmon and Markus Klein. Analyticity properties in scattering and spectral theory for Schrödinger operators with long-range radial potentials. Duke Math. J., 68(2):337-399, 1992.

[3] J. Dupont-Roc C. Cohen-Tannoudji and G. Gryndberg. Atom-Photon Interactions. Wiley - New York, 1992.

[4] A. Fring C. Figueira de Morisson Faria and R. Schrader. Analytical treatment of stabilization. arxiv:physics/9808047, 1998.

[5] S. L. Chin and P. Lambropoulos. Multiphoton Ionization of Atoms. New York:Academic Press, 1984.

[6] Michele Correggi, Gianfausto Dell'Antonio, Rodolfo Figari, and Andrea Mantile. Ionization for three dimensional time-dependent point interactions. Comm. Math. Phys., 257(1):169-192, 2005.

[7] O. Costin. Topological construction of transseries and introduction to generalized Borel summability. In Analyzable functions and applications, volume 373 of Contemp. Math., pages 137-175. Amer. Math. Soc., Providence, RI, 2005.

[8] O. Costin, R. D. Costin, and J. L. Lebowitz. Transition to the continuum of a particle in time-periodic potentials. In Advances in differential equations and mathematical physics (Birmingham, AL, 2002), volume 327 of Contemp. Math., pages 75-86. Amer. Math. Soc., Providence, RI, 2003.

[9] O. Costin, R. D. Costin, and J. L. Lebowitz. Time asymptotics of the Schrödinger wave function in time-periodic potentials. J. Statist. Phys., 116(1-4):283-310, 2004.

[10] O. Costin, R. D. Costin, J. L. Lebowitz, and A. Rokhlenko. Evolution of a model quantum system under time periodic forcing: conditions for complete ionization. Comm. Math. Phys., 221(1):1-26, 2001.

[11] O. Costin and A. Soffer. Resonance theory for Schrödinger operators. Comm. Math. Phys., 224(1):133-152, 2001. Dedicated to Joel L. Lebowitz.

[12] H. L. Cycon, R. G. Froese, W. Kirsch, and B. Simon. Schrödinger operators with application to quantum mechanics and global geometry. Texts and Monographs in Physics. Springer-Verlag, Berlin, 1987.

[13] Ingrid Daubechies. Ten lectures on wavelets, volume 61 of CBMS-NSF Regional Conference Series in Applied Mathematics. Society for Industrial and Applied Mathematics (SIAM), Philadelphia, PA, 1992. 
[14] G. F. Dell'Antonio, R. Figari, and A. Teta. The Schrödinger equation with moving point interactions in three dimensions. In Stochastic processes, physics and geometry: new interplays, I (Leipzig, 1999), volume 28 of CMS Conf. Proc., pages 99-113. Amer. Math. Soc., Providence, RI, 2000.

[15] J. Ecalle. Fonctions Resurgentes. Publications Mathematiques D'Orsay, 1981.

[16] A. Fring, V. Kostrykin, and R. Schrader. Ionization probabilities through ultra-intense fields in the extreme limit. J. Phys. A, 30(24):8599-8610, 1997.

[17] S Geltman. Multiphoton ionization of atoms. J. Phys. B: At. Mol. Phys., 10:831, 1974.

[18] Sandro Graffi and Kenji Yajima. Exterior complex scaling and the ac-stark effect in a coulomb field. Commun. Math. Phys., 89:277-301, 1983.

[19] T. Halfmann, L. P. Yatsenko, M. Shapiro, B. W. Shore, and K. Bergmann. Population trapping and laser-induced continuum structure in helium: Experiment and theory. Phys. Rev. A., 58(1):R46-R49, 1998.

[20] T. Kato. Perturbation Theory for Linear Operators, volume 132 of A Series of Comprehensive Studies in Mathematics. Springer Verlag, Berlin Heidelberg, 1976. Corrected Printing of the Second Edition.

[21] V. Kostrykin and R. Schrader. Ionization of atoms and molecules by short, strong laser pulses. J. Phys. A, 30(1):265-275, 1997.

[22] Jeffrey L. Krause, Kenneth J. Schafer, and Kenneth C. Kulander. Highorder harmonic generation from atoms and ions in the high intensity regime. Phys. Rev. Lett., 68(24):3535-3538, Jun 1992.

[23] M. Lewenstein, Ph. Balcou, M. Yu. Ivanov, Anne L'Huillier, and P. B. Corkum. Theory of high-harmonic generation by low-frequency laser fields. Phys. Rev. A, 49(3):2117-2132, Mar 1994.

[24] P. D. Miller, A. Soffer, and M. I. Weinstein. Metastability of breather modes of time-dependent potentials. Nonlinearity, 13(3):507-568, 2000.

[25] S.C. Cowley R.M. Susskind and E.J. Valeo. Multiphoton ionization in a short range potential: A nonperturbative approach. Phys. Rev. A, 42:3090, 1990.

[26] A. Rokhlenko, O. Costin, and J. L. Lebowitz. Decay versus survival of a localized state subjected to harmonic forcing: exact results. J. Phys. A, 35(42):8943-8951, 2002.

[27] C. Rothe, S. I. Hintschich, and A. P. Monkman. Violation of the exponential-decay law at long times. Physical Review Letters, 96(16):163601, 2006. 
[28] A. Soffer and M. I. Weinstein. Time dependent resonance theory. Geom. Funct. Anal., 8(6):1086-1128, 1998.

[29] M. Stein and Rami Shakarchi. Complex Analysis. Princeton University Press, 2003.

[30] L.P. Yatsenko T. Peters and T. Halfmann. Experimental demonstration of selective coherent population transfer via a continuum. Phys. Rev. Let., 95(10), 2005.

[31] K. Yajima. Resonances for the ac-stark effect. Commun. Math. Phys., 87:331-352, 1982. 


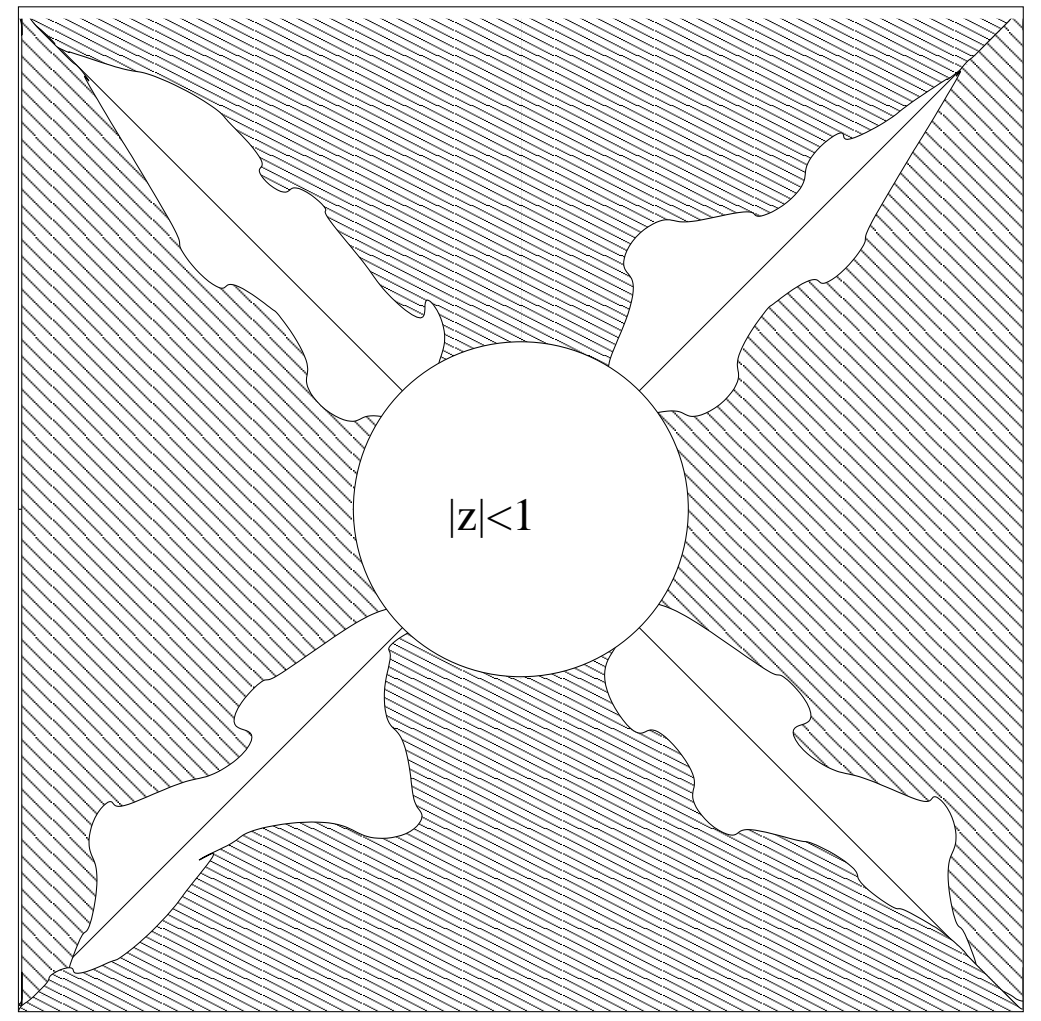

Figure 1: Schematic diagram of the sectors used in the proof of theorem 1 for the special case $N=2$. The shaded regions indicate the sets $S^{+}$and $S^{-}$. Note that $S^{+}$and $S^{-}$nearly fill out and encompass the whole sector as $|z|$ becomes larger. 